\title{
HIGH-RESOLUTION NONOSCILLATORY CENTRAL SCHEMES WITH NONSTAGGERED GRIDS FOR HYPERBOLIC CONSERVATION LAWS*
}

\author{
G.-S. JIANG ${ }^{\dagger}$, D. LEVY ${ }^{\S}$, C.-T. LIN $\ddagger$, S. OSHER ${ }^{\ddagger}$, AND E. TADMOR $₫$ \\ Dedicated to our friend and colleague Ami Harten, notre Ami
}

\begin{abstract}
We present a general procedure to convert schemes which are based on staggered spatial grids into nonstaggered schemes. This procedure is then used to construct a new family of nonstaggered, central schemes for hyperbolic conservation laws by converting the family of staggered central schemes recently introduced in [H. Nessyahu and E. Tadmor, J. Comput. Phys., 87 (1990), pp. 408-463; X. D. Liu and E. Tadmor, Numer. Math., 79 (1998), pp. 397-425; G. S. Jiang and E. Tadmor, SIAM J. Sci. Comput., 19 (1998), pp. 1892-1917]. These new nonstaggered central schemes retain the desirable properties of simplicity and high resolution, and in particular, they yield Riemann-solver-free recipes which avoid dimensional splitting. Most important, the new central schemes avoid staggered grids and hence are simpler to implement in frameworks which involve complex geometries and boundary conditions.
\end{abstract}

Key words. hyperbolic conservation laws, central schemes, staggered grids

AMS subject classifications. Primary, 65M10; Secondary, 65M05

PII. S0036142997317560

1. Introduction. We introduce new nonoscillatory central schemes for the approximate solution of nonlinear systems of hyperbolic conservation laws. The new feature of our schemes is the use of regular, nonstaggered spatial grids. Consequently, by avoiding staggered grids, which are often encountered in central recipes, our new central schemes offer further simplicity without sacrificing high resolution. Here is a brief overview.

Many of the modern high-resolution approximations for nonlinear conservation laws (and related equations) employ the Godunov approach. To this end, the approximate solution is realized by a piecewise polynomial which is reconstructed from the evolving cell-averages. In this context, we may distinguish between the two main classes of Godunov methods: upwind and central schemes.

Godunov's original scheme [3] is the forerunner of all upwind schemes. Its higherorder and multidimensional generalizations were constructed, analyzed, and implemented with great success during the 1970s and 1980s; consult [15,4] and the references therein. Upwind schemes evaluate their cell-averages over the same spatial cells at all time steps. This in turn requires characteristic information along the discontinuous interfaces of these spatial cells. It is the need to trace the characteristic fans - using approximate Riemann solvers, dimensional splitting, etc. - that greatly complicates the upwind algorithms.

\footnotetext{
* Received by the editors February 27, 1997; accepted for publication (in revised form) August 29, 1997; published electronically November 13, 1998. This research was supported by DARPA/ONR grant N00014-92-J-1890, ONR grant N00014-91-J-1076, NSF grant DMS97-06827, and the Sackler Institute for Scientific Computations at Tel-Aviv University.

http://www.siam.org/journals/sinum/35-6/31756.html

${ }^{\dagger}$ Courant Institute of Mathematical Sciences, NYU, New York, NY 10012 (gsj@cims.nyu.edu).

‡Department of Mathematics, UCLA, Los Angeles, CA 90095 (clin@math.ucla.edu, sjo@math. ucla.edu, tadmor@math.ucla.edu).

$\S$ Départment de Mathématiques, Ecole Normale Supérieure, 45 rue d'Ulm, 75230 Paris Cedex 05, France (dlevy@dmi.ens.fr).

ISchool of Mathematical Sciences, Tel-Aviv University, Tel-Aviv 69978, Israel.
} 
The Lax-Friedrichs ( $\mathrm{LxF})$ scheme [2] is the other canonical first-order scheme, which is the forerunner of all central schemes. Like the Godunov scheme, it is based on piecewise-constant approximate solution; its Riemann-solver-free recipe, however, is considerably simpler. Unfortunately, the excessive numerical viscosity in the LxF scheme yields a relatively poor resolution, which seems to have delayed the development of a high-resolution central scheme, parallel to the earlier developments of high-resolution upwind schemes.

A second-order sequel to the LxF scheme was introduced by Nessyahu and Tadmor in [22]. (Another approach to second-order central differencing based on characteristics tracing was introduced by Sanders and Wieser in [25]). The Nessyahu-Tadmor (NT) scheme retains the advantage of a simple, Riemann-solver-free recipe, and at the same time it enjoys high resolution comparable to the upwind results. In a recent series of works, the NT scheme was extended to higher orders $[21,7]$ and several space dimensions $[1,9,30]$, as well as incompressible flows and more general geometries $[17,11,10]$.

The common feature of all of these NT central schemes is the evolution of cellaverages over staggered cells, that is, cells which alternate every other time step. The importance of staggering is due to the fact that cell interfaces are secured in neighborhoods around the smooth midcells of the previous time step. The main advantage is simplicity: the costly Riemann characteristic decompositions from the upwind framework are now replaced by straightforward componentwise quadratures; dimensional splitting errors are avoided. At the same time, the use of high-order nonoscillatory piecewise polynomials (which are reconstructed from the staggered cellaverages) retain high resolution comparable with the upwind results.

In this paper we present a general procedure to convert general central schemes based on staggered grids over regular, nonstaggered grids. To convert into a nonstaggered formulation, we reaverage the reconstructed values of the underlying staggered scheme. In this manner, we recover the cell-averages of the central scheme over the original, nonstaggered grid cells. The whole procedure can be written in a two-step predictor-corrector method which totally avoids staggering.

When implemented in the context of the staggered NT schemes mentioned above, our procedure yields a new family of nonoscillatory, high-resolution central schemes based on nonstaggered spatial grids. Here we retain the original high resolution without giving up simplicity. Indeed, as before, the recipe of our new nonstaggered central schemes is free of (approximate) Riemann solvers, dimensional splitting, etc. The main new feature, however, is the further simplicity offered by a nonstaggered recipe, since one avoids the need to alternate between two staggered grids, which is particularly cumbersome near the boundaries.

The paper is organized as follows: In section 2 we motivate our procedure in the context of the first-order LxF scheme, resulting in the so called modified Lax-Friedrichs scheme. In section 3 we proceed with the second- and third-order NT schemes $[22,21]$, and in section 4 we deal with the multidimensional NT central scheme $[9,1]$.

In section 5 we present numerical simulations in which we implemented the methods of sections 3 and 4 on several nontrivial one- and two-dimensional problems. These simulations show that the results obtained with our new nonstaggered versions of the central schemes are comparable with previous results obtained using the staggered versions. It is remarkable that by using such simple, Riemann-solver-free algorithms, one is able to obtain the approximate solution of complex one- and two-dimensional problems with such high resolution. In this respect, the central schemes reported in 
this paper offer a versatile computational procedure for a large variety of nonlinear convective problems. Conclusions are in section 6 .

Finally, we would like to refer to the recent work of Liu and Osher [20] as another alternative recipe of the Riemann-solver-free central scheme. Here one employs a modified version of the pointwise essentially nonoscillatory (ENO) reconstruction introduced in [26], combined with the weighted ENO scheme devised in [8], and the Shu-Osher Runge-Kutta integration [26]. In the second-order case, the modified reconstruction resembles the second-order NT scheme. The result is a simple and uniformly high-order central scheme which, like the nonstaggered schemes reported in this paper, does not require field-by-field decomposition, staggered grids, etc.

2. Motivation: The first-order $\mathbf{L x F}$ scheme. In this section we present our general mechanism for transforming staggered schemes into nonstaggered schemes. We focus on numerical methods for hyperbolic conservation laws but in no way limit ourselves to this framework. First, we demonstrate these ideas for the onedimensional, first-order LxF scheme. We then apply in sections 3 and 4 the same principles to high-order schemes and to higher dimensions.

We approximate solutions to the one-dimensional hyperbolic system of conservation laws

$$
u_{t}+f(u)_{x}=0
$$

subject to the prescribed initial data, $u(x, t=0)=u_{0}(x)$. For simplicity, we assume an equally spaced grid, $\Delta x=x_{j+1}-x_{j}$, and abbreviate by $\bar{w}_{j}^{n}$ the approximate cellaverage at time $t=t^{n}$, associated with the cell $I_{j}:=\left\{\left|x-x_{j}\right| \leq \frac{\Delta x}{2}\right\}$. We also denote by $\chi_{j}(x)$ the characteristic function of the cell $I_{j}$, i.e., $\chi_{j}(x):=1_{I_{j}}$.

The staggered $\mathrm{LxF}$ approximation to (2.1) can be derived, e.g., by constructing a piecewise-constant interpolant through the given cell-averages, $w\left(x, t^{n}\right)=\sum_{j} \bar{w}_{j}^{n} \chi_{j}(x)$. This interpolant is then evolved exactly in time according to the conservation law (2.1), and finally projected onto the staggered cell-averages at the next time level. For $\Delta t \max _{u}\left|f^{\prime}(u)\right| \leq \frac{\Delta x}{2}$, the exact solution of (2.1) remains smooth at the integer grid points, $x_{j-1}, x_{j}$. Consequently, the required integrals of the fluxes are evaluated in terms of their constant initial data, without using any (approximate) Riemann solvers. The difference between the staggered cell-averages, $\bar{w}_{j+\frac{1}{2}}^{n+1}-\bar{w}_{j+\frac{1}{2}}^{n}$, equals the difference between the averages of the fluxes over the staggered control-volume, and hence, the LxF scheme amounts to

$$
\bar{w}_{j+\frac{1}{2}}^{n+1}=\frac{1}{2}\left(\bar{w}_{j}^{n}+\bar{w}_{j+1}^{n}\right)-\lambda\left[f\left(w_{j+1}^{n}\right)-f\left(w_{j}^{n}\right)\right], \quad w_{j}:=w\left(x_{j}, t^{n}\right)=\bar{w}_{j} .
$$

To eliminate the staggering, without losing the simplicity, we can define, e.g., the nonstaggered cell-average at time $t^{n+1}, \bar{w}_{j}^{n+1}$, as the average of its two neighboring staggered cell-averages,

$$
\begin{aligned}
\bar{w}_{j}^{n+1} & :=\frac{\bar{w}_{j-\frac{1}{2}}^{n+1}+\bar{w}_{j+\frac{1}{2}}^{n+1}}{2} \\
& =\frac{1}{4}\left(\bar{w}_{j-1}^{n}+2 \bar{w}_{j}^{n}+\bar{w}_{j+1}^{n}\right)-\frac{\lambda}{2}\left[f\left(w_{j+1}^{n}\right)-f\left(w_{j-1}^{n}\right)\right], \quad w_{j}=\bar{w}_{j} .
\end{aligned}
$$

Analogously, this nonstaggered cell-average, $\bar{w}_{j}^{n+1}$, can be defined by using a double nonstaggered control volume to formulate the scheme (see the right-hand side 

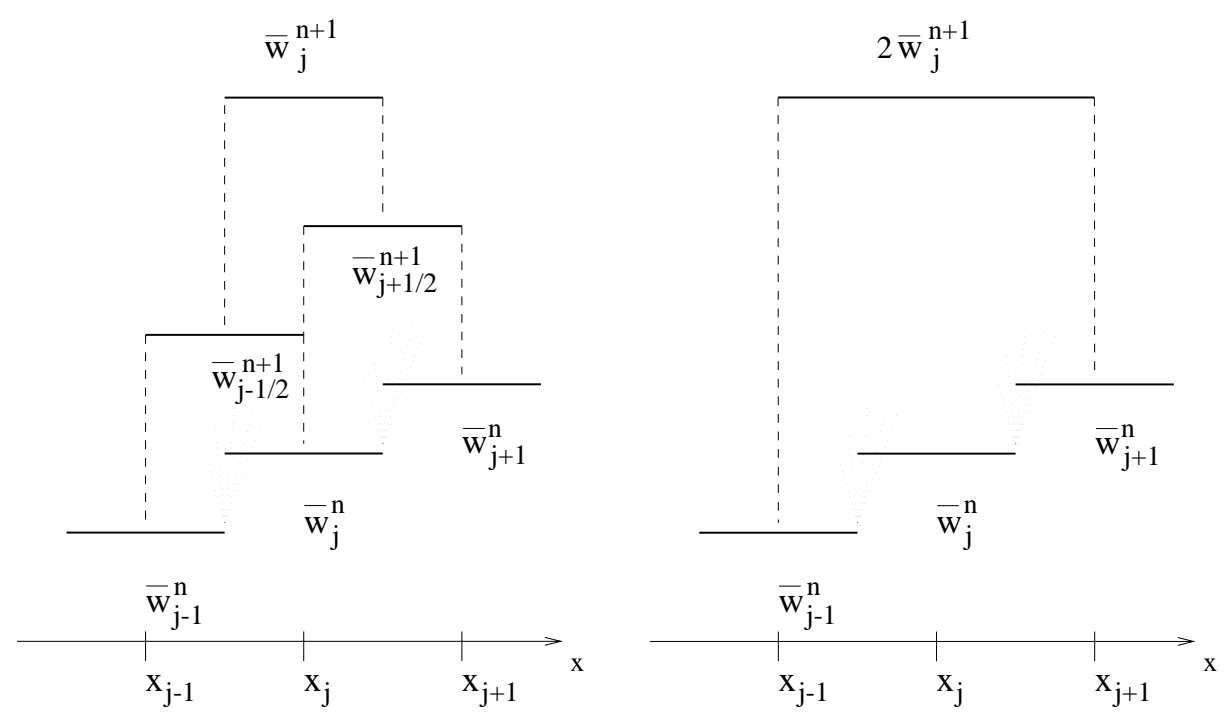

FIG. 2.1. Modified LxF scheme.

(RHS) of Figure 2.1). $\bar{w}_{j}^{n+1}$ is then defined as half of the value obtained at time $t^{n+1}$. This procedure is equivalent to the direct averaging presented in (2.3). The main limitation of these averaging approaches is that they are both first order and hence cannot be extended to higher-order approximations. This leads us to a third and final procedure.

We reconstruct a piecewise-constant reconstruction from the calculated staggered cell-averages at time $t^{n+1}$ (consult the left-hand side (LHS) of Figure 2.1),

$$
w\left(x, t^{n+1}\right)=\sum_{j} p_{j+\frac{1}{2}}^{n+1} \chi_{j+\frac{1}{2}}, \quad p_{j+\frac{1}{2}}^{n+1}=\bar{w}_{j+\frac{1}{2}}^{n+1} .
$$

This interpolant is then projected on the nonstaggered cell-averages to define

$$
\bar{w}_{j}^{n+1}:=\frac{1}{\Delta x}\left[\int_{x_{j-\frac{1}{2}}}^{x_{j}} w+\int_{x_{j}}^{x_{j+\frac{1}{2}}} w\right]=\frac{1}{\Delta x}\left[\int_{x_{j-\frac{1}{2}}}^{x_{j}} \bar{w}_{j-\frac{1}{2}}^{n+1}+\int_{x_{j}}^{x_{j+\frac{1}{2}}} \bar{w}_{j+\frac{1}{2}}^{n+1}\right],
$$

which is equivalent to (2.3). The advantage of this approach is that it can be easily extended for high-order accurate schemes. We note in passing that this modified version of the (first-order) LxF scheme is already known in the literature (e.g., see [29]).

To summarize, in order to transform a staggered scheme into a nonstaggered scheme, one has to reconstruct a piecewise-polynomial interpolant from the staggered cell-averages and project it on the nonstaggered cell-averages. We are now ready to implement this idea to construct nonstaggered versions of the high-order staggered schemes.

\section{One-dimensional extensions to higher orders.}

3.1. The second-order NT scheme. We follow the prototype LxF example in section 2 to construct a nonstaggered scheme from the staggered second-order NT scheme in [22]. The NT scheme is based on reconstructing a piecewise-linear (MUSCL- 
type) interpolant from the known staggered cell-averages at time $t^{n}$,

$$
w\left(x, t^{n}\right)=\sum_{j}\left[\bar{w}_{j}^{n}+w_{j}^{\prime}\left(\frac{x-x_{j}}{\Delta x}\right)\right] \chi_{j}(x) .
$$

Here and below, $w_{j}^{\prime}$ denotes the discrete slopes. A possible computation of these slopes, which results in an overall nonoscillatory scheme (consult [22]), is given by the family of discrete derivatives parameterized with $1 \leq \theta \leq 2$; that is, for any grid function $\left\{v_{j}\right\}$ we set

$$
v_{j}^{\prime}=M M \theta\left\{v_{j-1}, v_{j}, v_{j+1}\right\}:=M M\left(\theta \Delta v_{j+\frac{1}{2}}, \frac{1}{2}\left(\Delta v_{j-\frac{1}{2}}+\Delta v_{j+\frac{1}{2}}\right), \theta \Delta v_{j-\frac{1}{2}}\right) .
$$

Here, $\Delta$ denotes the centered differencing, $\Delta v_{j+1 / 2}=v_{j+1}-v_{j}$, and $M M$ denotes the min-mod nonlinear limiter

$$
M M\left\{x_{1}, x_{2}, \ldots\right\}= \begin{cases}\min _{j}\left\{x_{j}\right\} & \text { if } x_{j}>0 \quad \forall j \\ \max _{j}\left\{x_{j}\right\} & \text { if } x_{j}<0 \quad \forall j, \\ 0 & \text { otherwise. }\end{cases}
$$

This interpolant, (3.1), is then evolved exactly in time and projected on the staggered cell-averages on the next time step, $t^{n+1}$, resulting in the two-step predictorcorrector form,

$$
\begin{aligned}
& w_{j}^{n+\frac{1}{2}}=w_{j}^{n}-\frac{\lambda}{2} f_{j}^{\prime}, \quad w_{j}:=w\left(x_{j}, t^{n}\right)=\bar{w}_{j} \\
& \bar{w}_{j+\frac{1}{2}}^{n+1}=\frac{1}{2}\left(\bar{w}_{j}^{n}+\bar{w}_{j+1}^{n}\right)+\frac{1}{8}\left(w_{j}^{\prime}-w_{j+1}^{\prime}\right)-\lambda\left[f\left(w_{j+1}^{n+\frac{1}{2}}\right)-f\left(w_{j}^{n+\frac{1}{2}}\right)\right] .
\end{aligned}
$$

The discrete derivatives of the flux, $f_{j}^{\prime}$, can be computed, e.g., by $f_{j}^{\prime}=A_{j}^{n} w_{j}^{\prime}$, with $A_{j}^{n}:=A\left(\bar{w}_{j}^{n}\right)=f_{u}\left(\bar{w}_{j}^{n}\right)$; alternatively, one can apply the min-mod limiter to each of the components of $f$. This componentwise approach is one of the main advantages offered by the central NT schemes over the corresponding characteristic decompositions required by upwind schemes (consult the discussion in $[22,9]$ ).

In order to transform the staggered second-order scheme (3.4)-(3.5) into a nonstaggered scheme, we apply the method described in section 2 . First, we reconstruct a piecewise-linear interpolant through the calculated staggered cell-averages at time $t^{n+1}$ (consult Figure 3.1):

$$
w_{j \pm \frac{1}{2}}^{n+1}=\bar{w}_{j \pm \frac{1}{2}}^{n+1}+w_{j \pm \frac{1}{2}}^{\prime}\left(\frac{x-x_{j \pm \frac{1}{2}}}{\Delta x}\right) .
$$

The staggered discrete derivatives, $w_{j \pm \frac{1}{2}}^{\prime}$, are given by

$$
w_{j-\frac{1}{2}}^{\prime}=M M\left(\Delta \bar{w}_{j}^{n+1}, \Delta \bar{w}_{j-1}^{n+1}\right), \quad w_{j+\frac{1}{2}}^{\prime}=M M\left(\Delta \bar{w}_{j+1}^{n+1}, \Delta \bar{w}_{j}^{n+1}\right),
$$

with

$$
\begin{aligned}
\Delta \bar{w}_{i}^{n+1}:= & \bar{w}_{i+\frac{1}{2}}^{n+1}-\bar{w}_{i-\frac{1}{2}}^{n+1} \\
= & \frac{\bar{w}_{i+1}^{n}-\bar{w}_{i-1}^{n}}{2}-\frac{1}{8}\left(w_{i-1}^{\prime}-2 w_{i}^{\prime}+w_{i+1}^{\prime}\right) \\
& -\lambda\left[f\left(w_{i-1}^{n+\frac{1}{2}}\right)-2 f\left(w_{i}^{n+\frac{1}{2}}\right)+f\left(w_{i+1}^{n+\frac{1}{2}}\right)\right], \quad i=j-1, j, j+1 .
\end{aligned}
$$




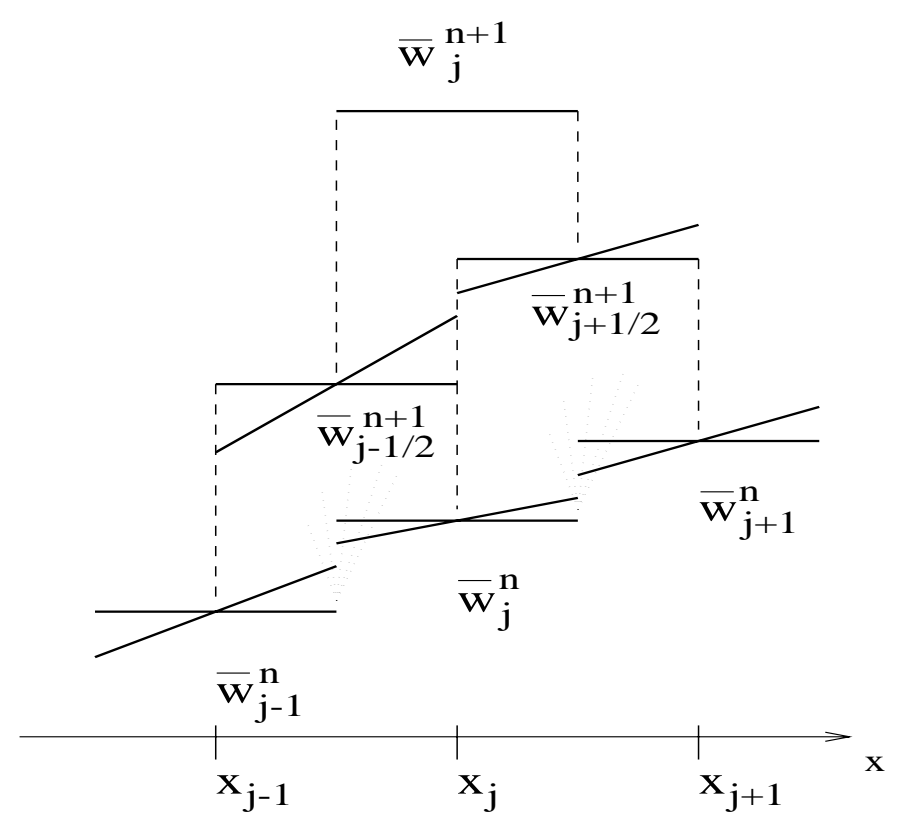

FIG. 3.1. Second-order reconstruction.

Second, the cell-averages at the next time step, $\bar{w}_{j}^{n+1}$, are obtained by averaging this interpolant, (3.6), resulting in the nonstaggered corrector scheme

$$
\begin{aligned}
\bar{w}_{j}^{n+1}= & \frac{1}{\Delta x}\left[\int_{x_{j-\frac{1}{2}}}^{x_{j}} w_{j-\frac{1}{2}}^{n+1}+\int_{x_{j}}^{x_{j+\frac{1}{2}}} w_{j+\frac{1}{2}}^{n+1}\right] \\
= & \frac{\bar{w}_{j-\frac{1}{2}}^{n+1}+\bar{w}_{j+\frac{1}{2}}^{n+1}}{2}-\frac{1}{8}\left(w_{j+\frac{1}{2}}^{\prime}-w_{j-\frac{1}{2}}^{\prime}\right) \\
= & \frac{1}{4}\left(\bar{w}_{j-1}^{n}+2 \bar{w}_{j}^{n}+\bar{w}_{j+1}^{n}\right)-\frac{1}{16}\left(w_{j+1}^{\prime}-w_{j-1}^{\prime}\right) \\
& -\frac{\lambda}{2}\left[f\left(w_{j+1}^{n+\frac{1}{2}}\right)-f\left(w_{j-1}^{n+\frac{1}{2}}\right)\right]-\frac{1}{8}\left(w_{j+\frac{1}{2}}^{\prime}-w_{j-\frac{1}{2}}^{\prime}\right) .
\end{aligned}
$$

Here, $\left\{w_{j}^{\prime}\right\}$ and $\left\{w_{j \pm \frac{1}{2}}^{\prime}\right\}$ are, respectively, the discrete derivatives at time levels $t^{n}$ and $t^{n+1}$ given in (3.2) and (3.7), and $\left\{w^{n+\frac{1}{2}}\right\}$ are predicted at time level $t^{n+\frac{1}{2}}$ according to $(3.4)$.

Remarks.

1. This nonstaggered second-order scheme (3.8) is still an essentially three-point scheme, since $\bar{w}_{j-1}^{n}=\bar{w}_{j}^{n}=\bar{w}_{j+1}^{n}$ implies $\bar{w}_{j \pm \frac{1}{2}}^{n} \equiv 0$, which, in turn, implies that $\Delta \bar{w}_{j}^{n+1} \equiv 0$ and hence $w_{j \pm \frac{1}{2}}^{\prime} \equiv 0$.

2. This procedure, for transforming the staggered cell-averages into nonstaggered cell-averages, can be reformulated in an ENO-like formulation. If we denote by $W^{n}(x)$ the primitive of $w^{n}(x)$, i.e., $W^{n}(x)=\int_{-\infty}^{x} w^{n}(\xi) d \xi$, then at time $t^{n+1}$ according to the staggering procedure, we actually calculate $W^{n+1}\left(x_{j}\right), j \in \mathbb{N}$. Utilizing a high-order ENO reconstruction, we interpo- 
late the known primitive values, such that $\forall x_{j} \leq x \leq x_{j+1}$,

$$
\begin{aligned}
& W^{n+1}(x):=W_{j}^{n+1}+\left(\frac{x-x_{j}}{\Delta x}\right)\left[W_{j+1}^{n+1}-W_{j}^{n+1}\right] \\
&+\frac{\left(x-x_{j}\right)\left(x-x_{j+1}\right)}{2(\Delta x)^{2}} \mathcal{M}\left[\Delta^{2}\left(W_{j-1}^{n+1}, W_{j}^{n+1}, W_{j+1}^{n+1}\right),\right. \\
&\left.\Delta^{2}\left(W_{j}^{n+1}, W_{j+1}^{n+1}, W_{j+2}^{n+1}\right)\right] .
\end{aligned}
$$

Here, $\Delta^{2}\left(W_{j-1}, W_{j}, W_{j+1}\right):=W_{j-1}-2 W_{j}+W_{j+1}$, and $\mathcal{M}$ denotes an ENO limiter given by

$$
\mathcal{M}\left\{x_{1}, x_{2}\right\}= \begin{cases}x_{1} & \text { if }\left|x_{1}\right| \leq\left|x_{2}\right| \\ x_{2} & \text { otherwise }\end{cases}
$$

Sampling (3.9) at the min-points, $x_{j+\frac{1}{2}}$, supplies the point values of the primitive, $W_{j+\frac{1}{2}}^{n+1}:=W^{n+1}\left(x_{j+\frac{1}{2}}\right)$, which are then used to define the nonstaggered cell-averages

$$
\bar{w}^{n+1}\left(x_{j}\right):=W_{j+\frac{1}{2}}^{n+1}-W_{j-\frac{1}{2}}^{n+1}, \quad j \in \mathbb{N} .
$$

If instead of ENO limiter $\mathcal{M}$ we use the min-mod limiter in (3.2), we end up with the familiar (3.8).

3. Other nonoscillatory limiters are also available. In particular, we mention here the nonoscillatory uniformly nonoscillatory (UNO) limiter [6]

$$
v_{j}^{\prime}=M M\left\{\Delta v_{j-\frac{1}{2}}+\frac{1}{2} M M\left(\Delta^{2} v_{j-1}, \Delta^{2} v_{j}\right), \Delta v_{j+\frac{1}{2}}-\frac{1}{2} M M\left(\Delta^{2} v_{j}, \Delta^{2} v_{j+1}\right)\right\} .
$$

3.2. A nonstaggered third-order scheme. The staggered third-order central scheme, presented by Liu and Tadmor in [21], is based on a nonoscillatory third-order reconstruction by Liu and Osher [19], $w\left(x, t^{n}\right)=\sum_{j} p_{j}(x) \chi_{j}(x)$. Each quadratic piece, $p_{j}(x)$, is of the form

$$
p_{j}(x)=w_{j}^{n}+w_{j}^{\prime}\left(\frac{x-x_{j}}{\Delta x}\right)+\frac{1}{2} w_{j}^{\prime \prime}\left(\frac{x-x_{j}}{\Delta x}\right)^{2} .
$$

Due to the conservation requirements, the reconstructed point values and discrete first and second derivatives are uniquely given by

$$
w_{j}:=\bar{w}_{j}-\frac{1}{24} w_{j}^{\prime \prime}, \quad w_{j}^{\prime}:=\theta_{j} \Delta_{0} \bar{w}_{j}^{n}, \quad w_{j}^{\prime \prime}:=\theta_{j} \Delta_{+} \Delta_{-} \bar{w}_{j}^{n} .
$$

$\theta_{j}$ is a nonlinear limiter, designed to prevent oscillations (for specific details on the construction of such a nonlinear limiter, consult [19] and [21]). Here, $w_{j}^{n}:=\bar{w}_{j}^{n}-\frac{w_{j}^{\prime \prime}}{24}$, are the reconstructed point values which, starting with the third-order reconstructions, are not necessarily equal to the cell-averages.

As in the first-order and second-order schemes, an exact evolution of this piecewisequadratic reconstruction, followed by a projection on the staggered averages, yields the scheme which can be formalized in terms of a predictor step

$$
w_{j}^{n+\alpha}=w_{j}^{n}+\lambda \alpha \dot{w}_{j}^{n}+\frac{(\lambda \alpha)^{2}}{2} \ddot{w}_{j}^{n}, \quad \alpha=\frac{1}{2}, 1,
$$


followed by the corrector

$$
\begin{aligned}
\bar{w}_{j+\frac{1}{2}}^{n+1}= & \frac{1}{2}\left(\bar{w}_{j}^{n}+\bar{w}_{j+1}^{n}\right)+\frac{1}{8}\left(w_{j}^{\prime}-w_{j+1}^{\prime}\right)-\frac{\lambda}{6}\left\{\left[f\left(w_{j+1}^{n}\right)+4 f\left(w_{j+1}^{n+\frac{1}{2}}\right)+f\left(w_{j+1}^{n+1}\right)\right]\right. \\
(3.13) & \left.-\left[f\left(w_{j}^{n}\right)+4 f\left(w_{j}^{n+\frac{1}{2}}\right)+f\left(w_{j}^{n+1}\right)\right]\right\} .
\end{aligned}
$$

The "dotted" notation, $\dot{w}_{j}^{n}, \ddot{w}_{j}^{n}$, abbreviates the first and the second time-derivatives, respectively. The construction of this scheme is based on a more accurate Taylor expansion to predict the mid-values, as well as Simpson's time-integration method, which provides the required accuracy in time.

It is a matter of applying the straightforward arguments to construct from this staggered version a nonstaggered version. From the staggered cell-averages, we reconstruct again a piecewise-quadratic reconstruction. This reconstruction is then projected on the nonstaggered averages, resulting in

$$
\bar{w}_{j}^{n+1}=\frac{\bar{w}_{j-\frac{1}{2}}^{n+1}+\bar{w}_{j+\frac{1}{2}}^{n+1}}{2}+\frac{1}{8}\left(w_{j-\frac{1}{2}}^{\prime}-w_{j+\frac{1}{2}}^{\prime}\right)=: \mathcal{I}_{1}+\mathcal{I}_{2} .
$$

The average of the staggered cell-averages, $\mathcal{I}_{1}$, equals

$$
\begin{aligned}
\mathcal{I}_{1}= & \frac{1}{4}\left(\bar{w}_{j-1}^{n}+2 \bar{w}_{j}^{n}+\bar{w}_{j+1}^{n}\right)+\frac{1}{16}\left(w_{j-1}^{\prime}-w_{j+1}^{\prime}\right) \\
& -\frac{\lambda}{12}\left[f_{j+1}^{n}+4 f_{j+1}^{n+\frac{1}{2}}+f_{j+1}^{n+1}-f_{j-1}^{n}-4 f_{j-1}^{n+\frac{1}{2}}-f_{j-1}^{n+1}\right] .
\end{aligned}
$$

As before, $\left\{w_{j}^{\prime}\right\}$ are evaluated at time level $t^{n}$, and the discrete derivatives of the staggered cell-averages required in $\mathcal{I}_{2}=\frac{1}{8}\left(w_{j-\frac{1}{2}}^{\prime}-w_{j+\frac{1}{2}}^{\prime}\right)$ are given by $w_{j \pm \frac{1}{2}}^{\prime}=$ $\theta_{j \pm \frac{1}{2}} \Delta_{0} \bar{w}_{j \pm \frac{1}{2}}^{n+1}$ and are evaluated at time level $t^{n+1}$.

3.3. Boundary conditions. Clearly, one of the main advantages of the nonstaggered schemes is that they are simpler to implement in frameworks which involve complex geometries and boundary conditions.

The issue of nonoscillatory boundary conditions in the context of staggered central schemes was systematically studied in [18]. It is straightforward to implement the ideas presented in [18] to the present nonstaggered framework.

Since this implementation is direct, we list below the main ingredients and leave the fine details to the enthusiastic reader. The resulting boundary computations are much simpler than those in [18].

First, one has to distinguish between inflow and outflow boundary cells. In inflow boundary cells, the point values at the boundary must be prescribed. Hence, in order to avoid spurious oscillations, a constant reconstruction at those boundary cells replaces the piecewise-linear interpolant there. This first-order reconstruction at the boundary does not disturb the second-order accuracy of the internal scheme.

At outflow boundary cells, one has to implement a piecewise-linear reconstruction. Here, following the natural direction of the flow, the slopes are computed by extrapolating the values from the interior of the domain up to its boundary.

This nonoscillatory boundary treatment can be naturally extended to systems and to higher spatial dimensions as well. 


\section{Extensions to two dimensions.}

4.1. The first-order LxF scheme. In the two-dimensional case, we approximate solutions to the two-dimensional hyperbolic system of conservation laws, given by

$$
u_{t}+f(u)_{x}+g(u)_{y}=0,
$$

subject to the given initial data, $u(x, y, t=0)=u_{0}(x, y)$. We denote by $\bar{w}_{j, k}^{n}$, the approximate cell-average at time $t=t^{n}$, associated with the cell $I_{j, k}:=I_{j} \times I_{k}$, centered around $\left(x_{j}=j \Delta x, y_{k}=k \Delta y\right)$.

The arguments we applied in the one-dimensional case can be easily extended to higher dimensions. This extension is straightforward and is based on the exact same procedure we introduced in section 2 , in which a nonstaggered scheme is created from a staggered scheme by averaging the interpolants we construct from the given staggered values. Our first two-dimensional example concerns the first-order LxF scheme.

In its staggered form, the LxF scheme supplies the cell-averages at time $t^{n+1}$, $\bar{w}_{j+\frac{1}{2}, k+\frac{1}{2}}^{n+1}$, from the given cell-averages at time $t^{n}, \bar{w}_{j, k}^{n}$,

$$
\begin{aligned}
\bar{w}_{j+\frac{1}{2}, k+\frac{1}{2}}^{n+1}= & \frac{1}{4}\left(\bar{w}_{j, k}^{n}+\bar{w}_{j+1, k}^{n}+\bar{w}_{j, k+1}^{n}+\bar{w}_{j+1, k+1}^{n}\right) \\
& -\frac{\lambda}{2}\left[f\left(w_{j+1, k}^{n}\right)-f\left(w_{j, k}^{n}\right)+f\left(w_{j+1, k+1}^{n}\right)-f\left(w_{j, k+1}^{n}\right)\right] \\
& -\frac{\mu}{2}\left[g\left(w_{j, k+1}^{n}\right)-g\left(w_{j, k}^{n}\right)+g\left(w_{j+1, k+1}^{n}\right)-g\left(w_{j+1, k}^{n}\right)\right] .
\end{aligned}
$$

Here and below, $\lambda:=\frac{\Delta t}{\Delta x}$ and $\mu:=\frac{\Delta t}{\Delta y}$ denote the fixed mesh-ratios in the $x$ - and the $y$-direction, respectively.

Nonstaggered cell-averages, $\bar{w}_{j, k}^{n+1}$, can be then derived from the staggered cellaverages, $\bar{w}_{j+\frac{1}{2}, k+\frac{1}{2}}^{n+1}$, by reconstructing a piecewise-constant two-dimensional interpolant through the staggered cell-averages, and averaged over the nonstaggered cells.

As in the one-dimensional setup, in this case this procedure is equivalent to the averaging (consult Figure 4.1)

$$
\bar{w}_{j, k}^{n+1}=\frac{1}{4}\left(\bar{w}_{j+\frac{1}{2}, k+\frac{1}{2}}^{n+1}+\bar{w}_{j-\frac{1}{2}, k+\frac{1}{2}}^{n+1}+\bar{w}_{j-\frac{1}{2}, k-\frac{1}{2}}^{n+1}+\bar{w}_{j+\frac{1}{2}, k-\frac{1}{2}}^{n+1}\right) .
$$

A straightforward calculation yields

$$
\begin{aligned}
\bar{w}_{j, k}^{n+1}= & \frac{1}{16}\left[\bar{w}_{j-1, k-1}^{n}+2 \bar{w}_{j-1, k}^{n}+\bar{w}_{j-1, k+1}^{n}+2 \bar{w}_{j, k-1}^{n}+4 \bar{w}_{j, k}^{n}\right. \\
& \left.+2 \bar{w}_{j, k+1}^{n}+\bar{w}_{j+1, k+1}^{n}+2 \bar{w}_{j+1, k}^{n}+\bar{w}_{j+1, k+1}^{n}\right] \\
- & \frac{\lambda}{2}\left\langle f\left(w_{j+1, .}^{n}\right)-f\left(w_{j, .}^{n}\right)\right\rangle_{k}-\frac{\mu}{2}\left\langle g\left(w_{\cdot, k+1}^{n}\right)-g\left(w_{\cdot, k}^{n}\right)\right\rangle_{j} .
\end{aligned}
$$

Here, we used the centered averaging notations

$$
\left\langle w_{j,}\right\rangle_{k}=\frac{w_{j, k-1}+w_{j, k+1}}{2}, \quad\left\langle w_{\cdot, k}\right\rangle_{j}=\frac{w_{j-1, k}+w_{j+1, k}}{2} .
$$




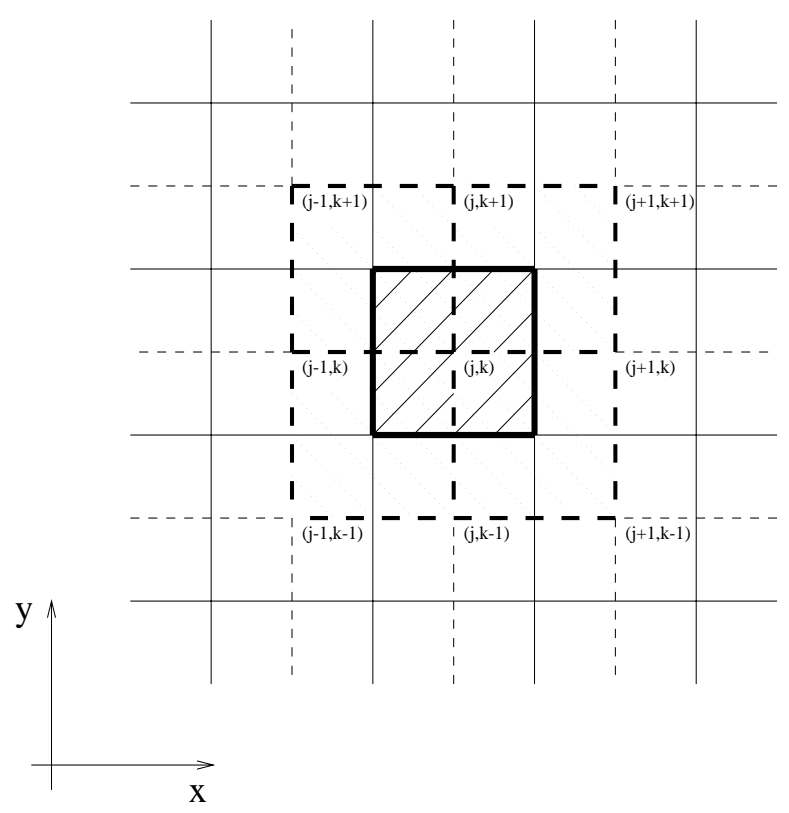

FIG. 4.1. Two-dimensional floor plan.

4.2. A second-order extension. A two-dimensional extension of the secondorder central NT scheme was introduced in $[9,1]$. As in the one-dimensional case, this staggered scheme can be viewed as an extension to the first-order LxF scheme. A piecewise-linear interpolant is reconstructed from the calculated cell-averages at time $t^{n}, w\left(x, y, t^{n}\right)=\sum_{j, k} p_{j, k}(x) \chi_{j, k}(x, y), \chi_{j, k}(x, y):=1_{I_{j, k}}$, where each linear piece, $p_{j, k}(x, y)$, is of the form

$$
p_{j, k}(x, y)=w_{j, k}^{n}+w_{j, k}^{\prime}\left(\frac{x-x_{j}}{\Delta x}\right)+w_{j, k}^{\prime}\left(\frac{y-y_{k}}{\Delta y}\right), \quad w_{j, k}=\bar{w}_{j, k} .
$$

Here and below, the prime and back-prime notations, $w_{j, k}^{\prime} \sim \Delta x \partial_{x} w, w_{j, k}^{\prime} \sim \Delta y \partial_{y} w$, denote the discrete derivatives in the $x$-direction and in the $y$-direction, respectively.

This reconstruction is then evolved in time and projected on the staggered cellaverages to yield the staggered cell-average, $\bar{w}_{j+\frac{1}{2}, k+\frac{1}{2}}^{n+1}$,

$$
\begin{aligned}
\bar{w}_{j+\frac{1}{2}, k+\frac{1}{2}}^{n+1}= & \frac{1}{4}\left(\bar{w}_{j, k}^{n}+\bar{w}_{j+1, k}^{n}+\bar{w}_{j, k+1}^{n}+\bar{w}_{j+1, k+1}^{n}\right) \\
& +\frac{1}{16}\left(w_{j, k}^{\prime}-w_{j+1, k}^{\prime}+w_{j, k+1}^{\prime}-w_{j+1, k+1}^{\prime}\right) \\
& +\frac{1}{16}\left(w_{j, k}^{\prime}-w_{j, k+1}^{\prime}+w_{j+1, k}^{\prime}-w_{j+1, k+1}^{\prime}\right) \\
& -\frac{\lambda}{2}\left[f\left(w_{j+1, k}^{n+\frac{1}{2}}\right)-f\left(w_{j, k}^{n+\frac{1}{2}}\right)+f\left(w_{j+1, k+1}^{n+\frac{1}{2}}\right)-f\left(w_{j, k+1}^{n+\frac{1}{2}}\right)\right] \\
& -\frac{\mu}{2}\left[g\left(w_{j, k+1}^{n+\frac{1}{2}}\right)-g\left(w_{j, k}^{n+\frac{1}{2}}\right)+g\left(w_{j+1, k+1}^{n+\frac{1}{2}}\right)-g\left(w_{j+1, k}^{n+\frac{1}{2}}\right)\right] .
\end{aligned}
$$

As before, the missing mid-values, $w^{n+\frac{1}{2}}$, are evaluated by Taylor's expansion

$$
w_{j, k}^{n+\frac{1}{2}}=\bar{w}_{j, k}-\frac{\lambda}{2} f_{j k}^{\prime}-\frac{\mu}{2} g_{j k}^{\prime} .
$$


In order to turn this staggered scheme into a nonstaggered scheme, we repeat our procedure by reconstructing a piecewise-linear interpolant through the staggered cellaverages given by (4.5),

$$
w_{j+\frac{1}{2}, k+\frac{1}{2}}^{n+1}=\bar{w}_{j+\frac{1}{2}, k+\frac{1}{2}}^{n+1}+w_{j+\frac{1}{2}, k+\frac{1}{2}}^{\prime}\left(\frac{x-x_{j+\frac{1}{2}, k+\frac{1}{2}}}{\Delta x}\right)+w_{j+\frac{1}{2}, k+\frac{1}{2}}^{\prime}\left(\frac{y-y_{j+\frac{1}{2}, k+\frac{1}{2}}}{\Delta y}\right) .
$$

This reconstruction is then averaged to yield the nonstaggered cell-average, $\bar{w}_{j, k}^{n+1}$, at time $t^{n+1}$ :

$$
\begin{aligned}
\bar{w}_{j, k}^{n+1}= & \frac{1}{4 \Delta x \Delta y}\left(\iint_{I_{j+\frac{1}{2}, k+\frac{1}{2}}} w_{j+\frac{1}{2}, k+\frac{1}{2}}^{n+1}+\iint_{I_{j-\frac{1}{2}, k+\frac{1}{2}}} w_{j-\frac{1}{2}, k+\frac{1}{2}}^{n+1}\right. \\
& \left.+\iint_{I_{j-\frac{1}{2}, k-\frac{1}{2}}} w_{j-\frac{1}{2}, k-\frac{1}{2}}^{n+1}+\iint_{I_{j+\frac{1}{2}, k-\frac{1}{2}}} w_{j+\frac{1}{2}, k-\frac{1}{2}}^{n+1}\right) \\
= & \frac{1}{4}\left(\bar{w}_{j+\frac{1}{2}, k+\frac{1}{2}}^{n+1}+\bar{w}_{j-\frac{1}{2}, k+\frac{1}{2}}^{n+1}+\bar{w}_{j-\frac{1}{2}, k-\frac{1}{2}}^{n+1}+\bar{w}_{j+\frac{1}{2}, k-\frac{1}{2}}^{n+1}\right) \\
& +\frac{1}{16}\left[\left(w_{j-\frac{1}{2}, k-\frac{1}{2}}^{\prime}-w_{j+\frac{1}{2}, k-\frac{1}{2}}^{\prime}\right)+\left(w_{j-\frac{1}{2}, k+\frac{1}{2}}^{\prime}-w_{j+\frac{1}{2}, k+\frac{1}{2}}^{\prime}\right)\right. \\
& \left.+\left(w_{j-\frac{1}{2}, k-\frac{1}{2}}^{\prime}-w_{j-\frac{1}{2}, k+\frac{1}{2}}^{\prime}\right)+\left(w_{j+\frac{1}{2}, k-\frac{1}{2}}^{\prime}-w_{j+\frac{1}{2}, k+\frac{1}{2}}^{\prime}\right)\right] .
\end{aligned}
$$

The staggered cell-averages, $\left\{\bar{w}_{j \pm \frac{1}{2}, k \pm \frac{1}{2}}^{n+1}\right\}$, are given by (4.5), while the discrete staggered derivatives, $\left\{w_{j \pm \frac{1}{2}, k \pm \frac{1}{2}}^{\prime}\right\}$, can be computed, e.g., using a min-mod limiter on the staggered cell-averages at time $t^{n+1}$.

Remark. The above method can be naturally extended to more accurate methods (such as the third-order two-dimensional scheme, introduced in [16]).

5. Numerical examples. In this section, we implement our second-order schemes presented in sections 3 and 4, for several one- and two-dimensional model problems. Below, we abbreviate the different schemes by NT- $p$ - $q$ : "NT" stands for the Nessyahu-Tadmor scheme (3.4)-(3.8); " $p$ " stands for the type of limiter used in computing the numerical derivatives ("MM1" and "MM2" refer to the $\theta$-dependent limiter (3.2) with $\theta=1$ and $\theta=2$, respectively; or the UNO limiter outlined in (3.10)); " $q$ " is either " $\mathrm{S}$ " (the staggered version) or "N" (the nonstaggered version).

Example 1. Accuracy test. In Table 1, we compare the accuracy of the nonstaggered schemes, NT-MM1-N, NT-MM2-N, and NT-UNO-N, with the accuracy of the corresponding staggered schemes, NT-MM1-S, NT-MM2-S, and NT-UNO-S. This accuracy check is done by approximating solutions to the linear problem, $u_{t}+u_{x}=0$, subject to the initial data $u(x, 0)=\sin 2 \pi x$. The accuracy of the computations was checked at time $T=1$, with $\mathrm{CFL}=0.475$.

These results show that the additional reconstruction step in the nonstaggered schemes results in slightly less accurate results when compared with the staggered schemes with the same limiter. The UNO limiter is the most accurate limiter from the three limiters we used, because it is the only limiter which is uniformly secondorder accurate. The penalty of using the UNO limiter is that it has a wider stencil (five points for computing numerical derivatives, whereas the MM limiter only utilizes three points). The computational cost with the UNO limiter, however, is almost the same as that with the MM2 limiter. The MM1 limiter is the most dissipative and hence the least accurate. 
TABLE 1 $T=1$.

Accuracy of staggered and nonstaggered schemes, $u_{t}+u_{x}=0, u(x, 0)=\sin 2 \pi x, C F L=0.475$,

\begin{tabular}{|c|c|c|c|c|c|c|c|c|}
\hline & \multicolumn{9}{|c|}{$N T-M M 1-S$} & \multicolumn{5}{c|}{$N T-M M 1-N$} \\
\hline$N$ & $L_{\infty}$ error & Order & $L_{1}$ error & Order & $L_{\infty}$ error & Order & $L_{1}$ error & Order \\
\hline 20 & $3.506 \mathrm{e}-2$ & - & $1.290 \mathrm{e}-2$ & - & $1.626 \mathrm{e}-1$ & - & $7.079 \mathrm{e}-2$ & - \\
40 & $1.380 \mathrm{e}-2$ & 1.35 & $3.166 \mathrm{e}-3$ & 2.03 & $7.151 \mathrm{e}-2$ & 1.19 & $2.863 \mathrm{e}-2$ & 1.31 \\
80 & $5.543 \mathrm{e}-3$ & 1.32 & $8.243 \mathrm{e}-4$ & 1.94 & $3.017 \mathrm{e}-2$ & 1.25 & $8.069 \mathrm{e}-3$ & 1.83 \\
160 & $2.251 \mathrm{e}-3$ & 1.30 & $2.065 \mathrm{e}-4$ & 2.00 & $1.243 \mathrm{e}-2$ & 1.28 & $2.173 \mathrm{e}-3$ & 1.89 \\
320 & $9.130 \mathrm{e}-4$ & 1.30 & $5.256 \mathrm{e}-5$ & 1.97 & $5.069 \mathrm{e}-3$ & 1.29 & $5.959 \mathrm{e}-4$ & 1.87 \\
\hline \multicolumn{7}{|c|}{$N T-M M 2-S$} & \\
\hline$N$ & $L_{\infty}$ error & Order & $L_{1}$ error & Order & $L_{\infty}$ error & Order & $L_{1}$ error & Order \\
\hline 20 & $1.474 \mathrm{e}-2$ & - & $5.735 \mathrm{e}-3$ & - & $6.903 \mathrm{e}-2$ & - & $2.410 \mathrm{e}-2$ & - \\
40 & $5.839 \mathrm{e}-3$ & 1.34 & $1.333 \mathrm{e}-3$ & 2.11 & $2.457 \mathrm{e}-2$ & 1.49 & $5.874 \mathrm{e}-3$ & 2.04 \\
80 & $2.121 \mathrm{e}-3$ & 1.46 & $3.107 \mathrm{e}-4$ & 2.10 & $8.691 \mathrm{e}-3$ & 1.50 & $1.328 \mathrm{e}-3$ & 2.15 \\
160 & $7.980 \mathrm{e}-4$ & 1.41 & $7.010 \mathrm{e}-5$ & 2.15 & $3.027 \mathrm{e}-3$ & 1.52 & $2.699 \mathrm{e}-4$ & 2.30 \\
320 & $3.365 \mathrm{e}-4$ & 1.25 & $1.580 \mathrm{e}-5$ & 2.15 & $1.041 \mathrm{e}-3$ & 1.54 & $5.497 \mathrm{e}-5$ & 2.30 \\
\hline \multicolumn{7}{|c|}{$N T-U N O-S$} & \multicolumn{7}{c|}{$N T-U N O-N$} & \\
\hline$N$ & $L_{\infty}$ error & Order & $L_{1}$ error & Order & $L_{\infty}$ error & Order & $L_{1}$ error & Order \\
\hline 20 & $2.887 \mathrm{e}-3$ & - & $1.867 \mathrm{e}-3$ & - & $1.115 \mathrm{e}-2$ & - & $7.201 \mathrm{e}-3$ & - \\
40 & $6.578 \mathrm{e}-4$ & 2.13 & $4.195 \mathrm{e}-4$ & 2.15 & $1.478 \mathrm{e}-3$ & 2.92 & $9.628 \mathrm{e}-4$ & 2.90 \\
80 & $1.616 \mathrm{e}-4$ & 2.03 & $1.029 \mathrm{e}-4$ & 2.03 & $2.336 \mathrm{e}-4$ & 2.66 & $1.486 \mathrm{e}-4$ & 2.70 \\
160 & $4.015 \mathrm{e}-5$ & 2.01 & $2.556 \mathrm{e}-5$ & 2.01 & $4.490 \mathrm{e}-5$ & 2.38 & $2.858 \mathrm{e}-5$ & 2.38 \\
320 & $9.909 \mathrm{e}-6$ & 2.02 & $6.308 \mathrm{e}-6$ & 2.02 & $1.024 \mathrm{e}-5$ & 2.13 & $6.516 \mathrm{e}-6$ & 2.13 \\
\hline
\end{tabular}

Example 2. Burgers's equation. Here, we approximate solutions to the inviscid Burgers's equation

$$
u_{t}+\left(\frac{u^{2}}{2}\right)_{x}=0, \quad-1 \leq x \leq 1,
$$

subject to the 2-periodic initial data $u_{0}(x)=0.25+0.5 \sin (\pi x)$. Figure 5.1 shows the solutions at time $T=1.1$, when the shock is well developed. The nonstaggered schemes smear the shock slightly more than the corresponding staggered schemes using the same limiter. In this problem, the MM2 limiter gives resolution similar to the UNO limiter.

Example 3. Buckley-Leverett problem. Here we apply the schemes to the BuckleyLeverett problem (whose flux is nonconvex),

$$
u_{t}+f(u)_{x}=0, \quad f(u)=\frac{u^{2}}{4 u^{2}+(1-u)^{2}},
$$

subject to the initial data $u_{0}(x)=1$ for $x \in[-0.5,0]$ and 0 otherwise. We compute the solution up to $T=0.4$ and use the CFL 0.475 . The results are presented in Figure 5.2. The MM2 limiter seems to give the wrong solution for both the staggered and the nonstaggered scheme. The UNO limiter, however, produces accurate results in both cases. Similar phenomena are found when the grid is refined and/or the CFL number is reduced. Nevertheless, in many other cases, we found that the MM2 and the UNO limiter produce rather similar results.

Example 4. One-dimensional Euler-Riemann problem. In this example, we apply the schemes to the one-dimensional Euler system,

$$
u_{t}+f(u)_{x}=0
$$




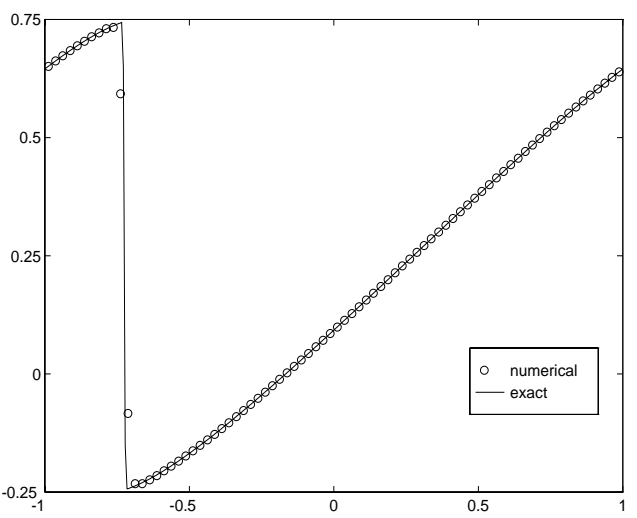

(a)

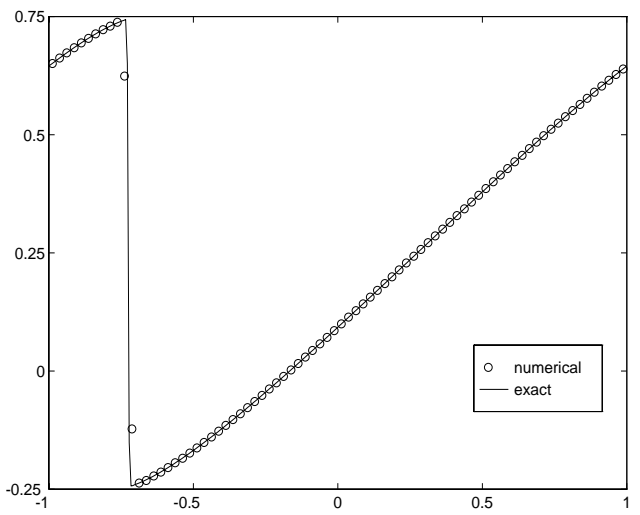

(b)

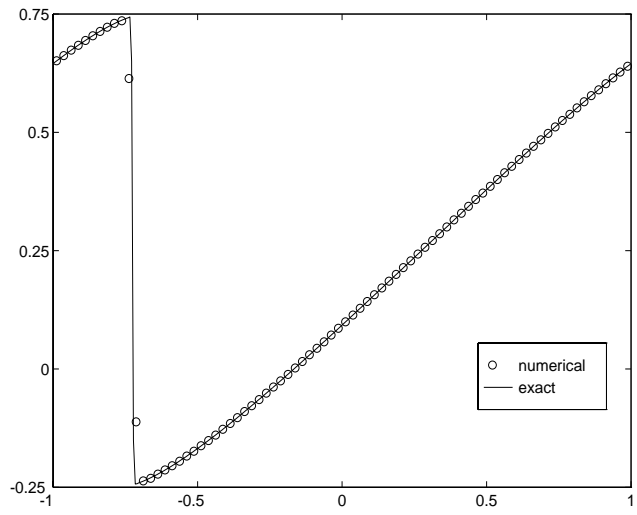

(c)

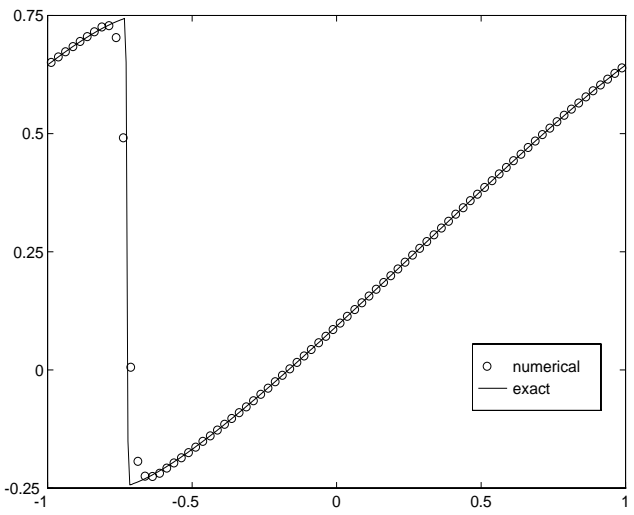

$\left(a^{\prime}\right)$

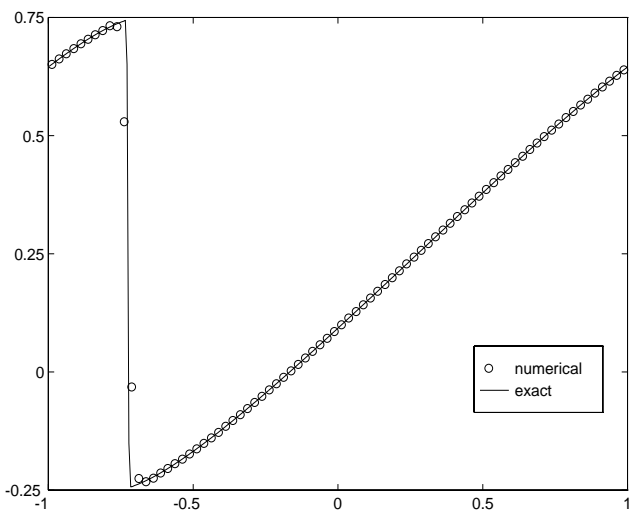

$\left(b^{\prime}\right)$

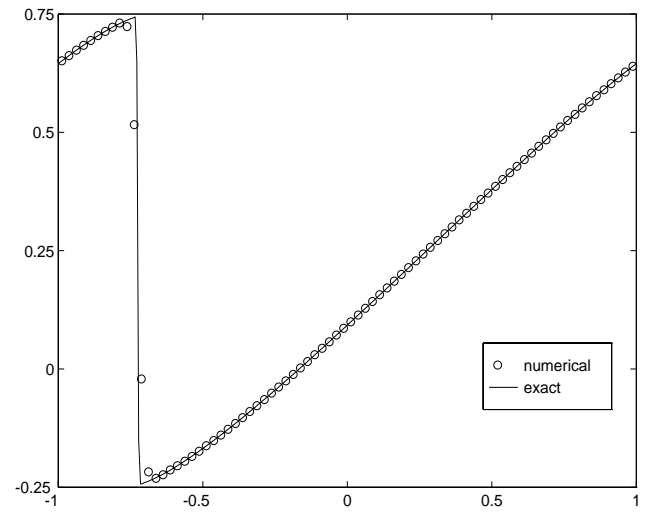

$\left(c^{\prime}\right)$

FIG. 5.1. One-dimensional Burgers's equation, $T=1.1, C F L=0.475, \Delta x=1 / 40$. (a) $N T$ $M M 1-S$; (a') NT-MM1-N; (b) NT-MM2-S; (b') NT-MM2-N; (c) NT-UNO-S; (c') NT-UNO-N.

where

$$
\begin{aligned}
u & =(\rho, \rho u, E)^{T} \\
f(u) & =\left(\rho u, P+\rho u^{2}, u(E+P)\right)^{T}
\end{aligned}
$$

augmented with $P=(\gamma-1)\left(E-\frac{1}{2} \rho u^{2}\right)$. Here $\rho, u, P$, and $E$ are, respectively, the 


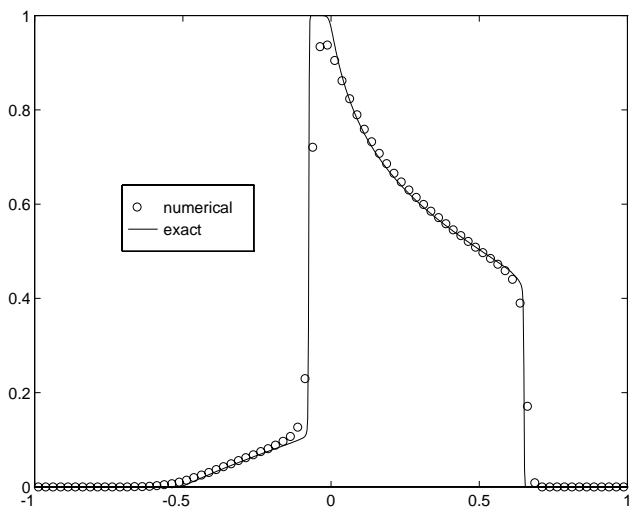

(a)

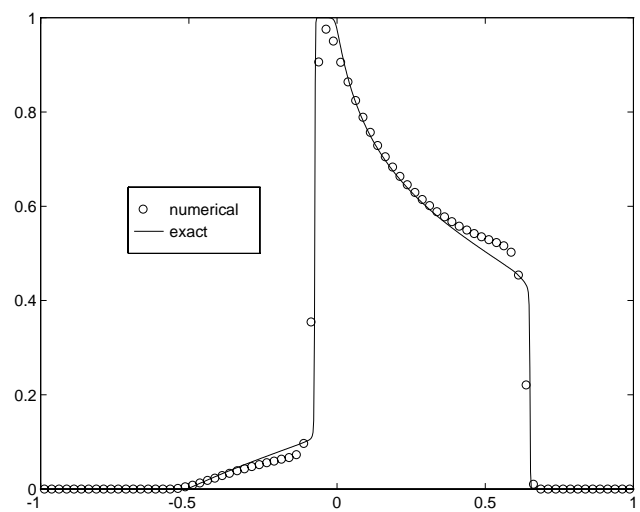

(b)

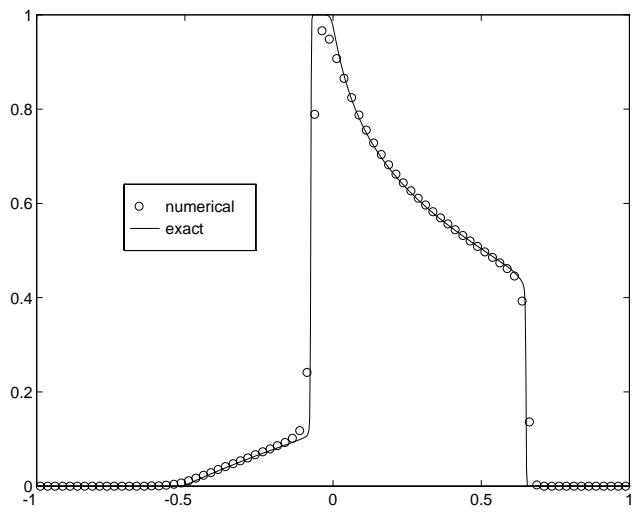

(c)

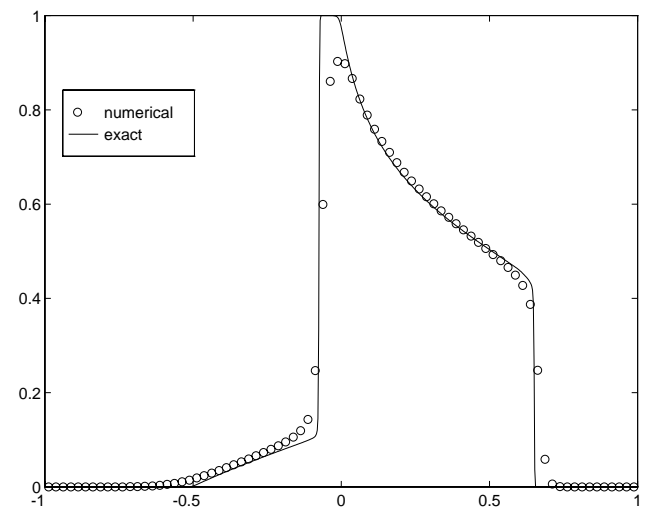

$\left(a^{\prime}\right)$

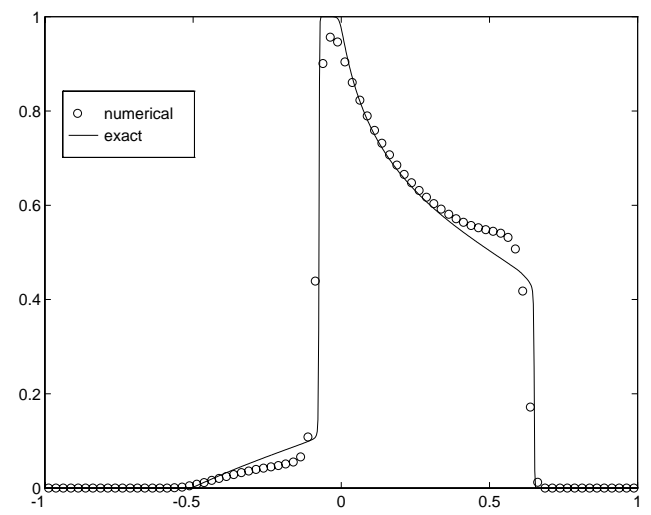

$\left(b^{\prime}\right)$

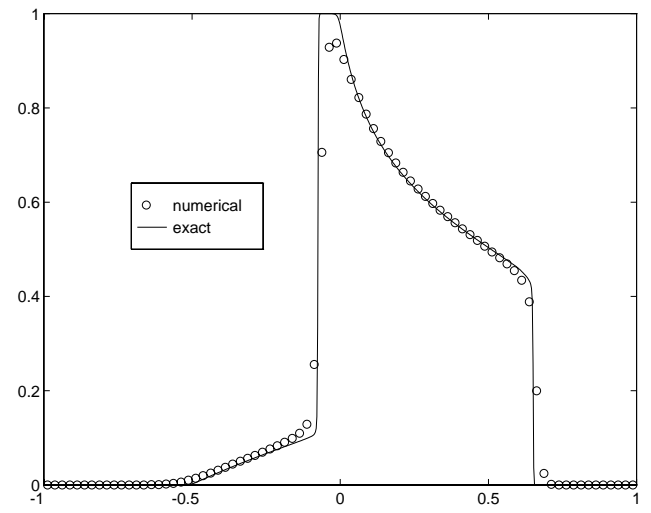

$\left(\mathrm{c}^{\prime}\right)$

FIG. 5.2. Buckley-Leverett problem. $T=0.4, C F L=0.475, \Delta x=1 / 40$. (a) NT-MM1-S; (a') $N T-M M 1-N$; (b) NT-MM2-S; (b') NT-MM2-N; (c) NT-UNO-S; (c') NT-UNO-N.

density, velocity, pressure, and total specific energy. The system is complemented with Riemann initial data of the form

$$
u(x, 0)= \begin{cases}u_{L} & \text { if } x<0 \\ u_{R} & \text { if } x>0\end{cases}
$$


- The first Riemann problem was proposed by Sod [27]. The initial data are taken as

$$
\left(\rho_{L}, u_{L}, P_{L}\right)=(1,0,1), \quad\left(\rho_{R}, u_{R}, P_{R}\right)=(0.125,0,0.1) .
$$

In Figure 5.3, we show the density, velocity, and pressure plots obtained by NT-UNO-S and NT-UNO-N. The schemes with the MM2 limiter produce similar results. Again, the nonstaggered scheme gives slightly lower resolution at the two ends of the rarefaction wave, the contact and the shock.

- The second Riemann problem was proposed by Lax [12]. Here, the initial data are given by

$$
\left(\rho_{L}, u_{L}, P_{L}\right)=(0.445,0.698,3.528), \quad\left(\rho_{R}, u_{R}, P_{R}\right)=(0.5,0,0.571) .
$$

We present the results by NT-UNO-S and NT-UNO-N in Figure 5.4. The MM2 limiter gives a slight overshoot in the density profile but otherwise is similar to the UNO limiter.

Example 5. Blast wave problem. In this example, we test the schemes on the blast wave problem which was carefully studied by Woodward and Colella [31]. The governing equation is the one-dimensional Euler system (5.3)-(5.4). The initial data are

$$
u(x, 0)= \begin{cases}u_{L} & \text { if } 0.0<x<0.1 \\ u_{M} & \text { if } 0.1<x<0.9 \\ u_{R} & \text { if } 0.9<x<1.0\end{cases}
$$

where

$$
\rho_{L}=\rho_{M}=\rho_{R}=1, \quad u_{L}=u_{M}=u_{R}=0, \quad P_{L}=10^{3}, P_{M}=10^{-2}, P_{R}=10^{2} .
$$

The reflective boundary condition is applied at both $x=0$ and $x=1$. The results of NT-UNO-S and NT-UNO-N are shown in Figure 5.5. The "exact" solution in the figures is obtained with a fourth-order ENO scheme on a very fine grid. Both schemes perform well at the strong shocks.

Example 6. Oblique Sod's problem. Here, we analyze the capability of the central schemes to resolve waves that are oblique to the computational mesh. The governing equation is the two-dimensional Euler system,

$$
u_{t}+f(u)_{x}+g(u)_{y}=0
$$

where

$$
\begin{aligned}
u & =(\rho, \rho u, \rho v, E)^{T}, \\
f(u) & =\left(\rho u, P+\rho u^{2}, \rho u v, u(E+P)\right)^{T}, \\
g(u) & =\left(\rho v, \rho u v, P+\rho v^{2}, v(E+P)\right)^{T}
\end{aligned}
$$

augmented with $P=(\gamma-1)\left(E-\frac{1}{2} \rho\left(u^{2}+v^{2}\right)\right)$. Here $\rho, u, v, P$, and $E$ are, respectively, the density, velocity in the $x$-direction, velocity in the $y$-direction, pressure, and total specific energy.

The two-dimensional Sod's problem is solved where the initial jump is at angle $\theta$ to the $x$-axis $\left(0<\theta \leq \frac{\pi}{2}\right)$. $\theta=\frac{\pi}{2}$ corresponds to the one-dimensional Sod's problem. If $0<\theta<\frac{\pi}{2}$, then all the produced waves are oblique to the rectangular 


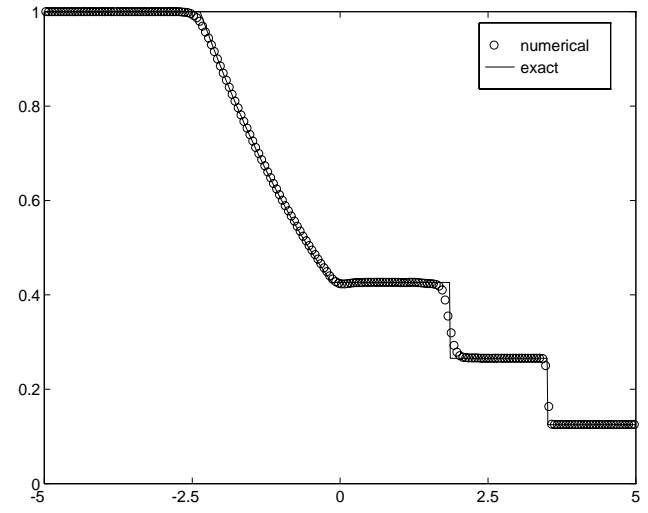

(a)

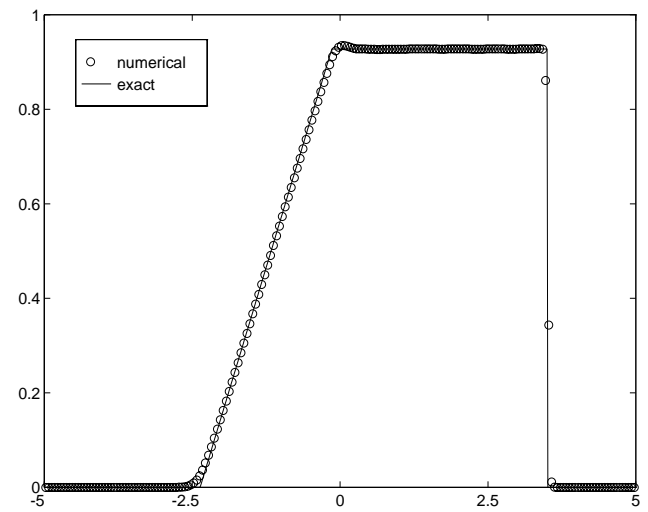

(b)

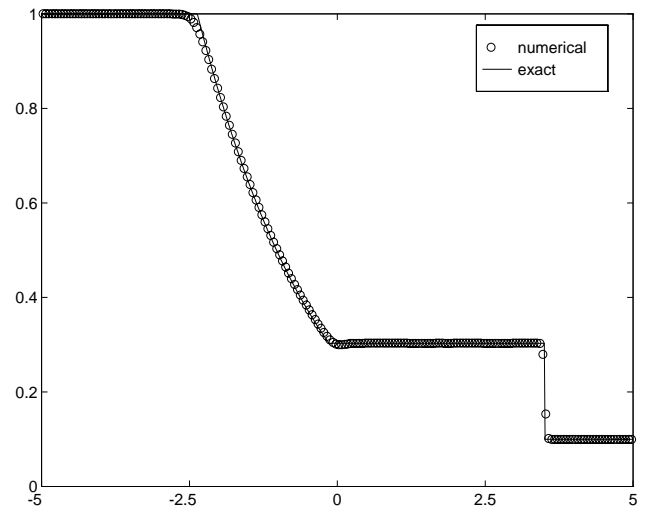

(c)

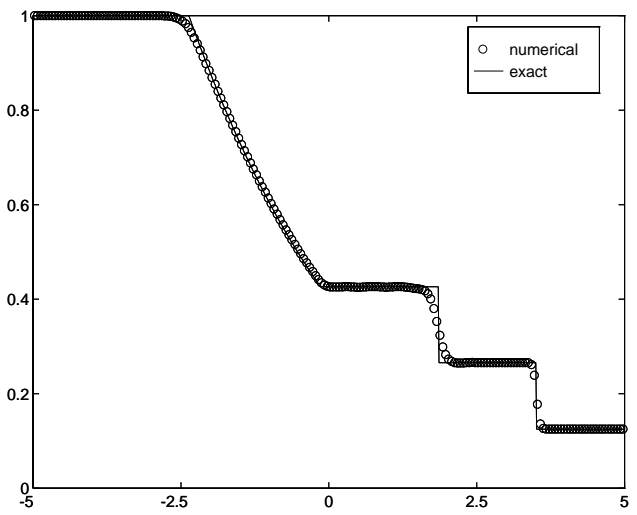

$\left(a^{\prime}\right)$

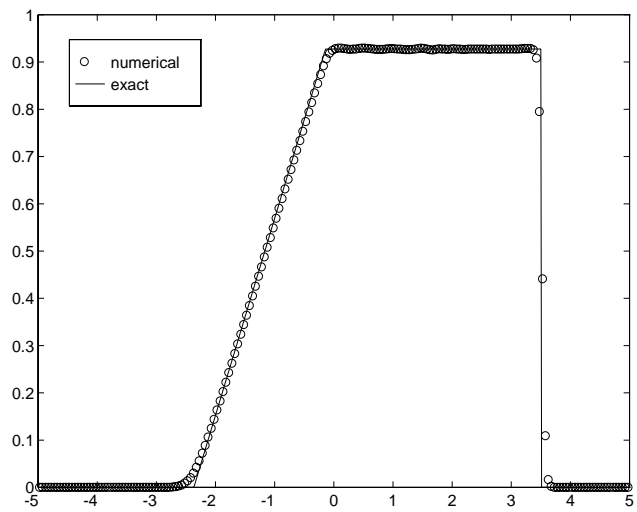

$\left(b^{\prime}\right)$

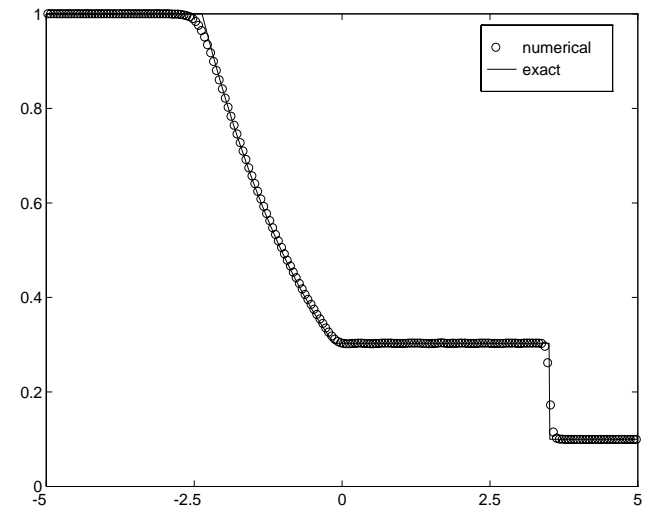

$\left(c^{\prime}\right)$

FIG. 5.3. Sod problem. $T=2.0, C F L=0.475, \Delta x=1 / 20$. (a), (b), (c) NT-UNO-S; $\left(\mathrm{a}^{\prime}\right),\left(\mathrm{b}^{\prime}\right)$,

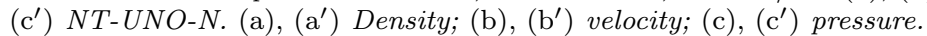

computational cell. The computational domain is $[0,6] \times[0,1]$ and the initial jump is positioned at $(x, y)=(2.25,0)$. The physical domain varies with $\theta$ and is taken as $\left[0, \frac{6}{\sin \theta}\right] \times\left[0, \frac{1}{\sin \theta}\right]$. The scaling factor, $\frac{1}{\sin \theta}$, supplies the same grid resolution normal to the wave propagation, on a given mesh and at a fixed time for every $\theta$. In the simulations, $\theta$ is taken as $\arctan 1, \arctan 2$, and $\arctan 4$. The solution is computed 


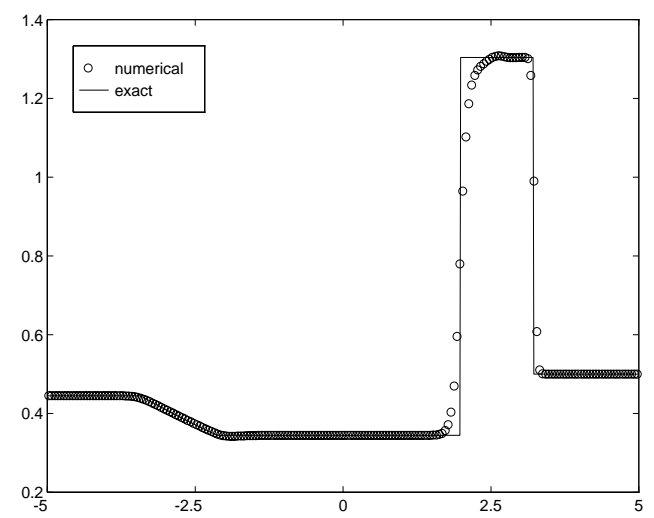

(a)

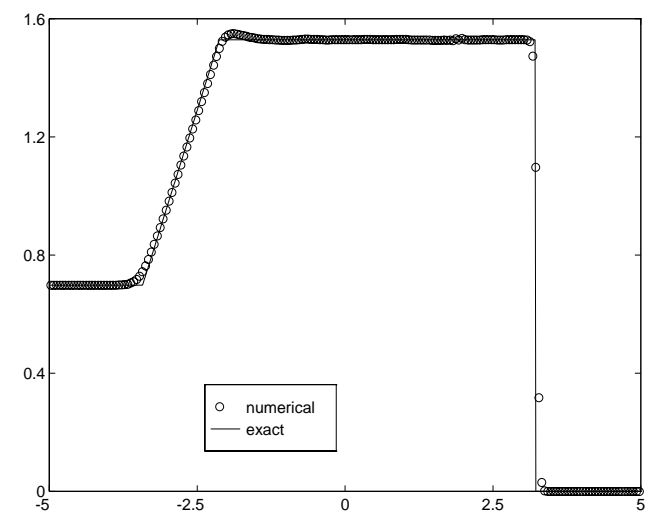

(b)

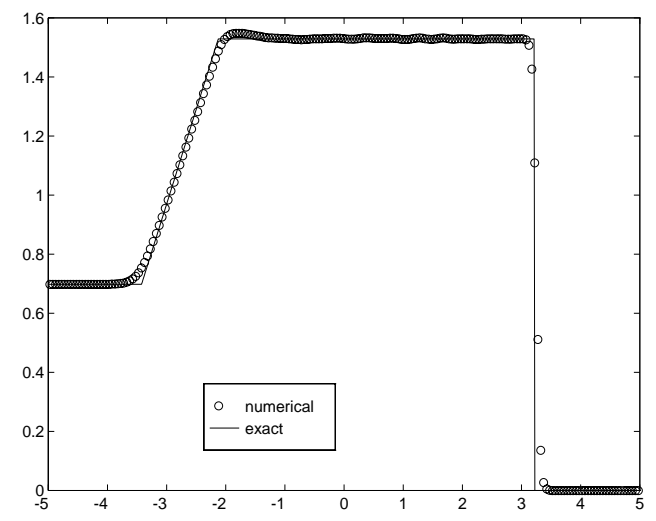

(a)

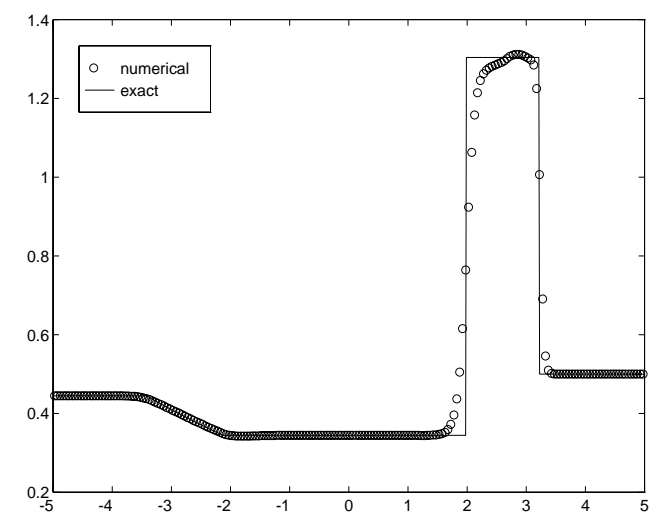

$\left(a^{\prime}\right)$

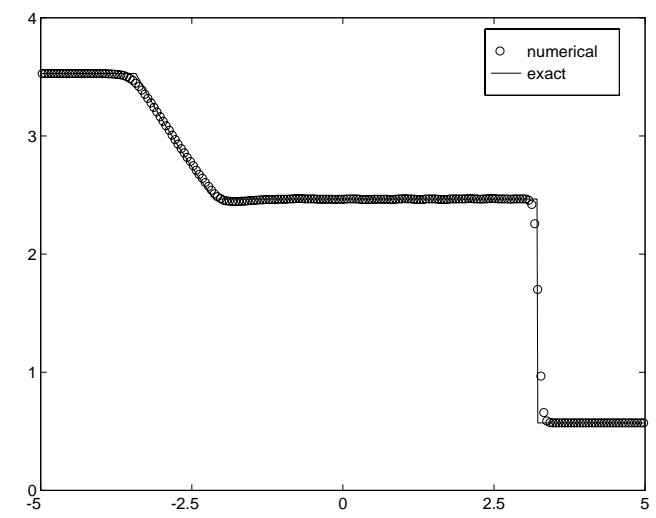

$\left(b^{\prime}\right)$

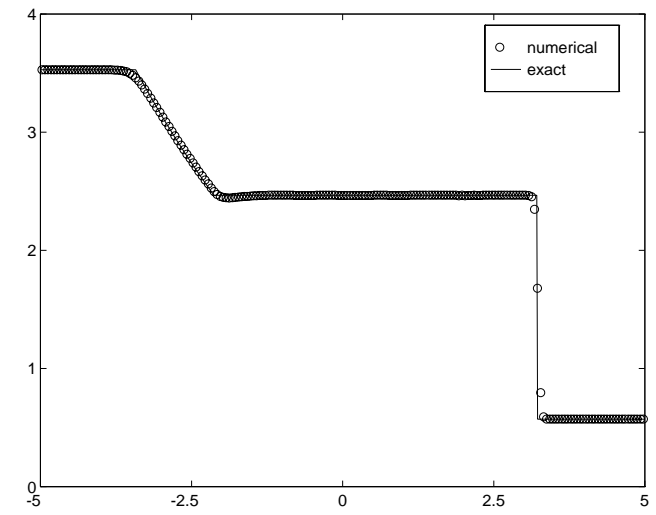

$\left(a^{\prime}\right)$

FIG. 5.4. Lax problem. $T=1.3, C F L=0.475, \Delta x=1 / 20$. (a), (b), (c) NT-UNO-S; $\left(\mathrm{a}^{\prime}\right),\left(\mathrm{b}^{\prime}\right)$,

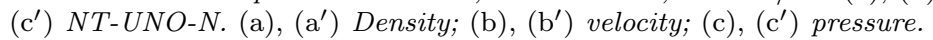

up to $T=1.2$ on a $192 \times 32$ grid; consult [8].

The results are shown in Figure 5.6. (a) and $\left(\mathrm{a}^{\prime}\right)$ present a one-dimensional cut along $y=0$. (a) is obtained by the staggered scheme, while $\left(\mathrm{a}^{\prime}\right)$ is obtained by the nonstaggered scheme. The MM2 limiter produces slight overshoots at the shock and the contact. It achieves, however, the same sharp resolution as the UNO limiter. In 


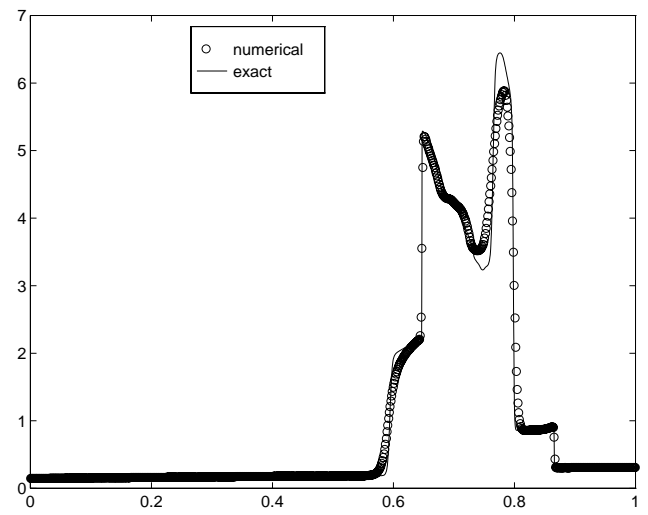

(a)

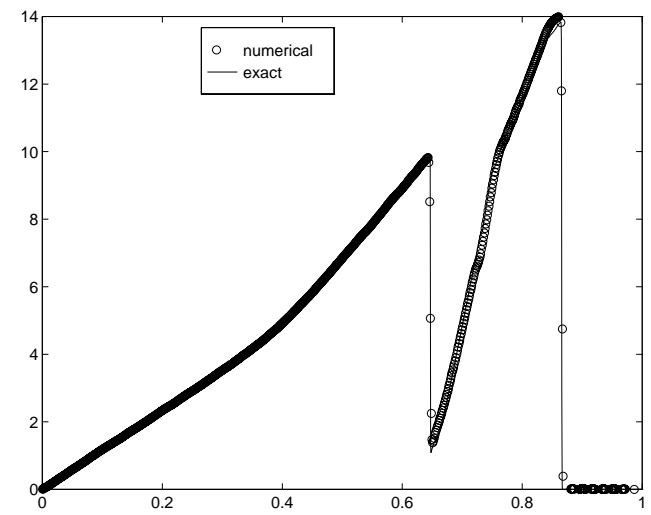

(b)

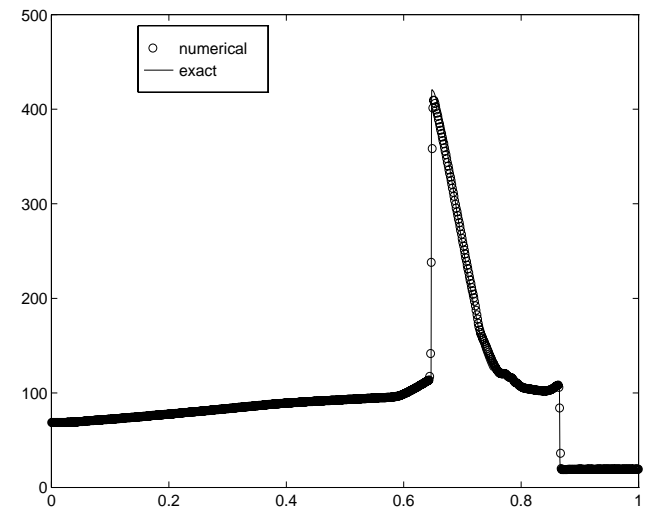

(c)

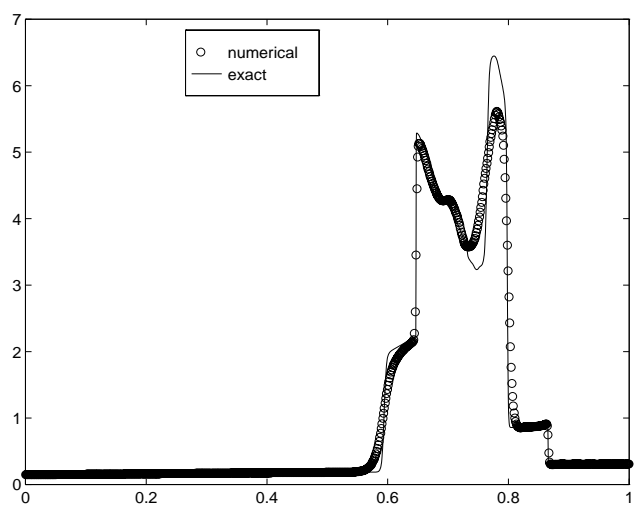

$\left(a^{\prime}\right)$

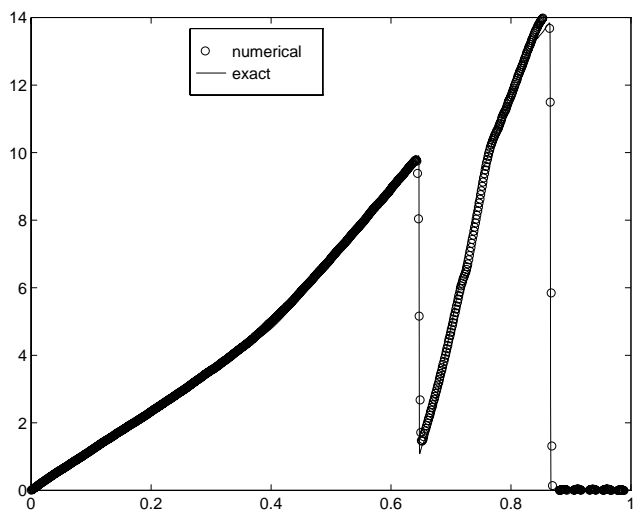

$\left(b^{\prime}\right)$

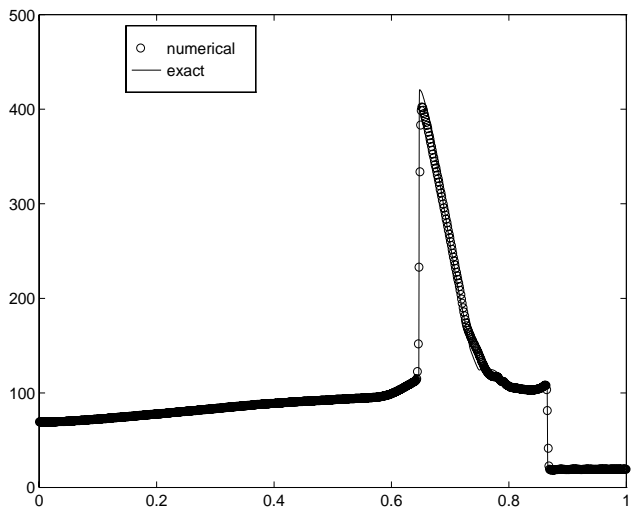

$\left(c^{\prime}\right)$

FIG. 5.5. Blast wave problem. $T=0.038, C F L=0.475, \Delta x=1 / 800$. (a), (b), (c) NT-UNO-S;

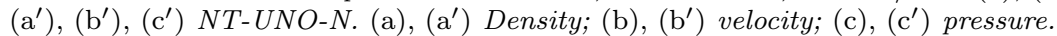

(b)-(d) and $\left(b^{\prime}\right)-\left(d^{\prime}\right)$, we plot, for each scheme, the differences between the densities at an oblique angle and for the one-dimensional case. The differences in density resolution at different angles are rather small for all schemes. The magnitude here is comparable to that reported by Jiang and Shu [8]. An interesting point, which may be typical to cell-average-type schemes for this problem, is that the largest difference 


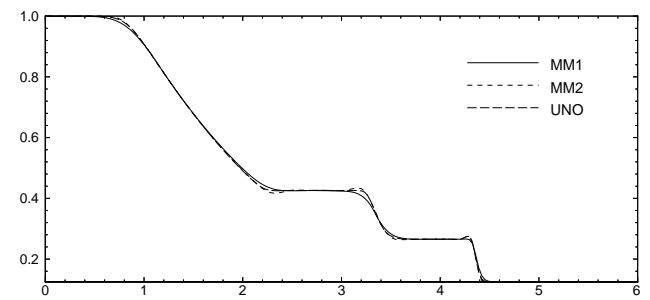

(a)

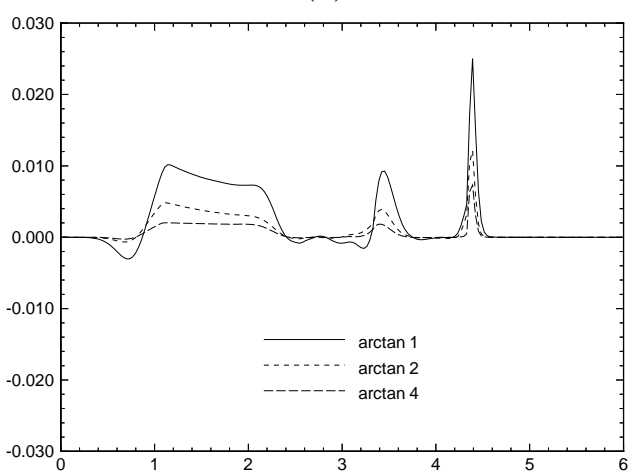

(b)

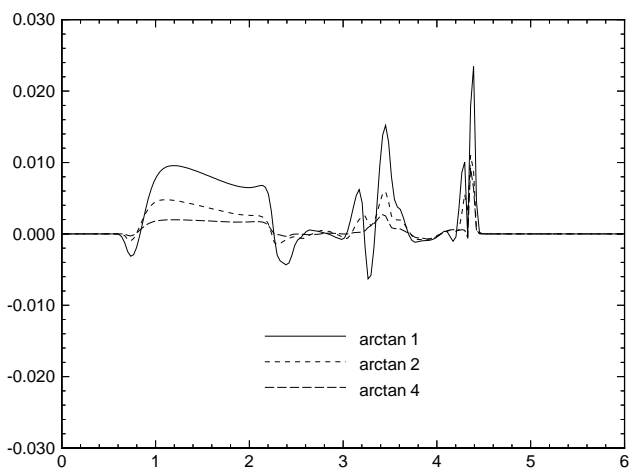

(c)

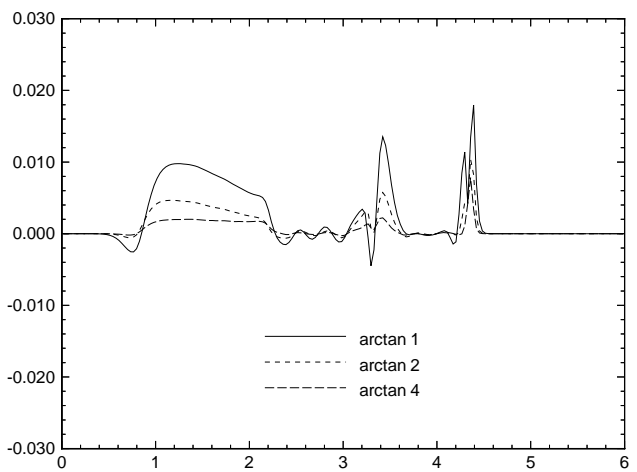

(d)

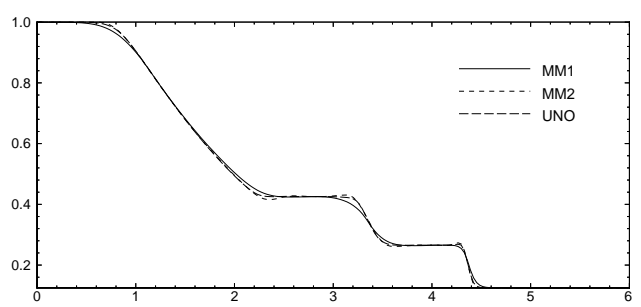

$\left(a^{\prime}\right)$

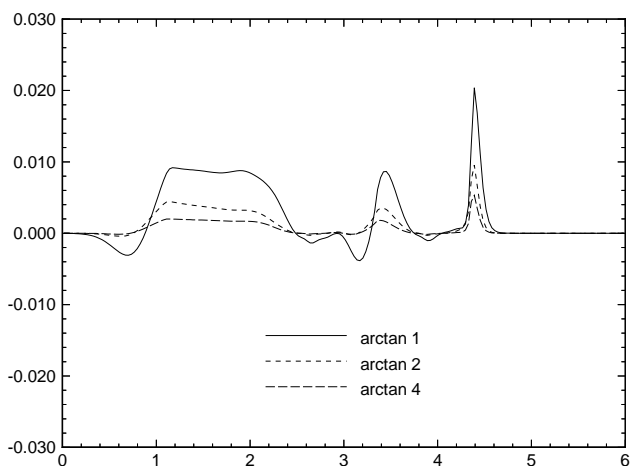

$\left(b^{\prime}\right)$

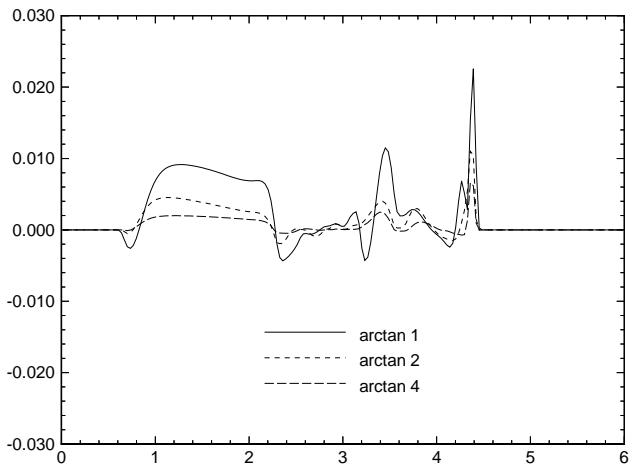

$\left(c^{\prime}\right)$

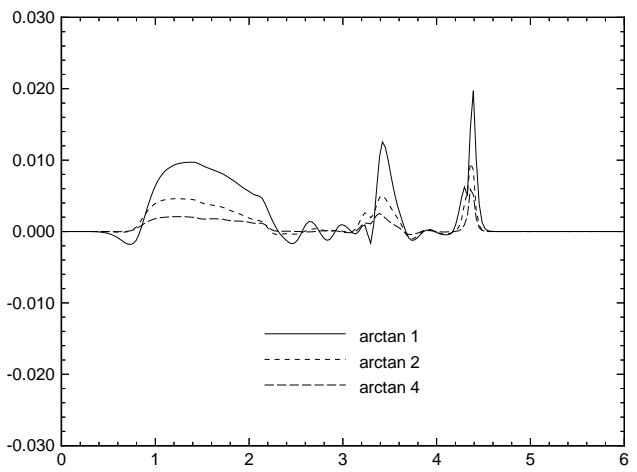

$\left(d^{\prime}\right)$

FIG. 5.6. Oblique Sod problem. $T=1.2, C F L=0.475, \Delta x=\Delta y=1 / 32$. (a) Staggered; $\left(\mathrm{a}^{\prime}\right)$ nonstaggered. (a), $\left(\mathrm{a}^{\prime}\right)$ Density at $y=0$ for $\theta=\arctan 1 .(\mathrm{b})-(\mathrm{d}),\left(\mathrm{b}^{\prime}\right)-\left(\mathrm{d}^{\prime}\right)$ Density difference at $y=0: \rho_{\theta}-\rho_{1 D}$ with $\theta=\arctan 1, \arctan 2, \arctan 4$. (b) NT-MM1-S; (b') NT-MM1-N; (c) NT-MM2-S; (c') NT-MM2-N; (d) NT-UNO-S; (d') NT-UNO-N. 


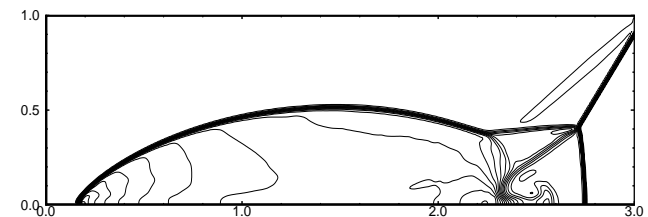

(a)

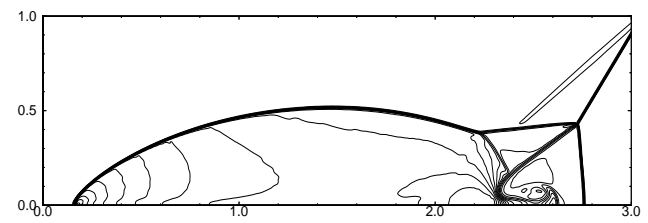

(b)

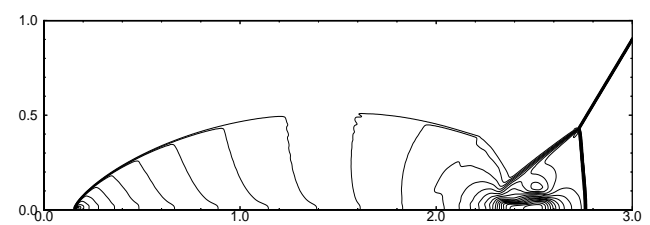

(c)

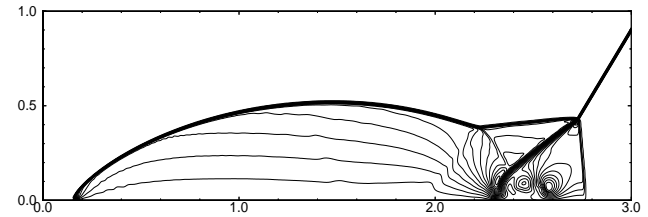

(d)

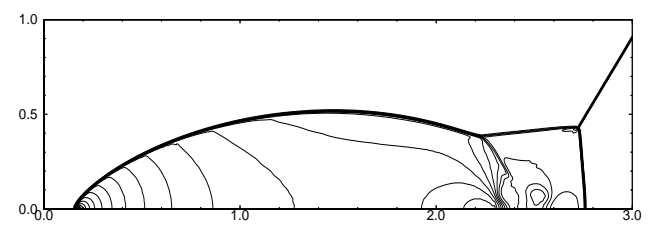

(e)

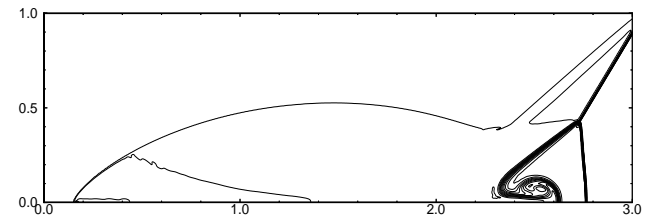

(f)

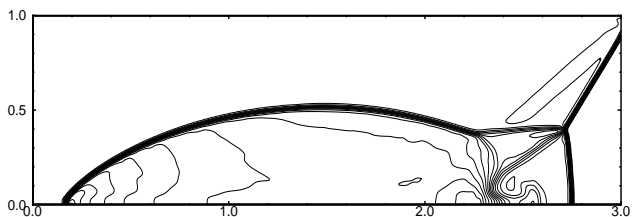

$\left(a^{\prime}\right)$

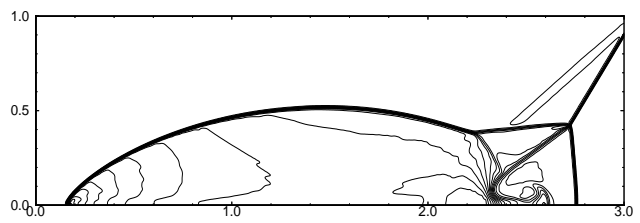

$\left(b^{\prime}\right)$

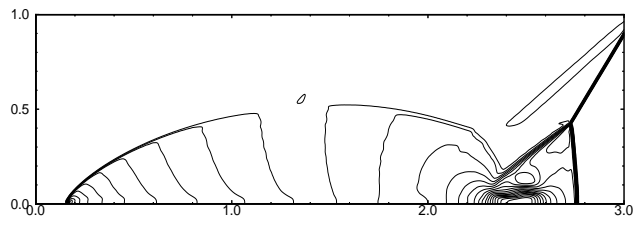

$\left(c^{\prime}\right)$

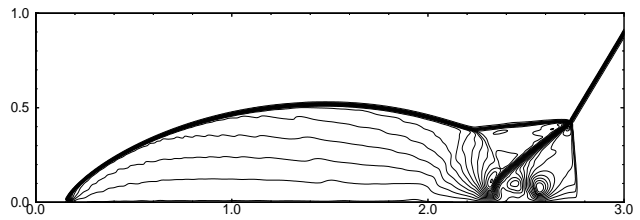

$\left(d^{\prime}\right)$

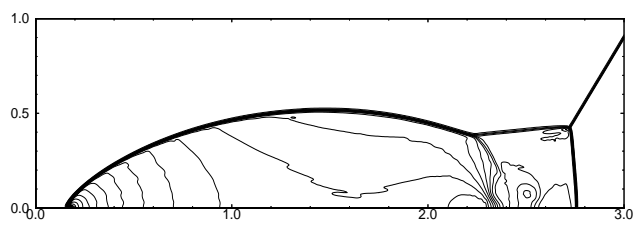

$\left(\mathrm{e}^{\prime}\right)$

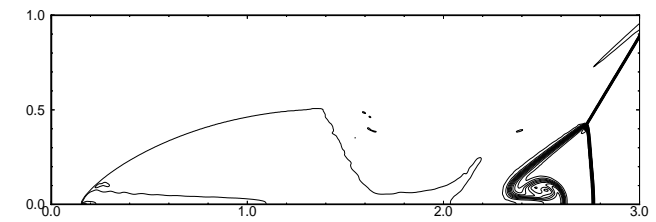

$\left(\mathrm{f}^{\prime}\right)$

Fig. 5.7. Double Mach reflection problem. $T=0.2, C F L=0.475$. Grid: (a), ( $\left.\mathrm{a}^{\prime}\right) 480 \times 120$;

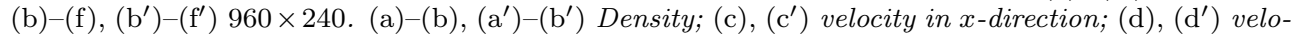

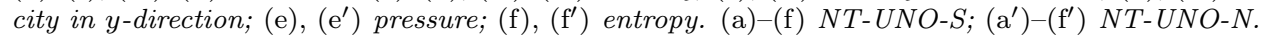

in density happens for the angle $\theta=\arctan 1$, (where the wave fronts are diagonal in the square cell used). The next largest difference is for $\theta=\arctan 2$, and the least difference is for $\theta=\arctan 4$. For finite difference schemes, it seems that the opposite is true; i.e., the magnitude of the difference increases as the angle changes from $\arctan 1$ to $\arctan 4$. 
Example 7. Double Mach reflection problem. The governing equation for this problem is the two-dimensional Euler system (5.5)-(5.6). The computational domain is $[0,4] \times[0,1]$. The reflecting wall lies at the bottom of the computational domain starting from $x=\frac{1}{6}$. Initially a right-moving Mach 10 shock is positioned at $(x, y)=$ $\left(\frac{1}{6}, 0\right)$ and makes a $60^{\circ}$ angle with the $x$-axis. For the bottom boundary, the exact postshock condition is imposed from $x=0$ to $x=\frac{1}{6}$, and a reflective boundary condition is used for the rest of the $x$-axis. At the top boundary of the computational domain, the data is set to describe the exact motion of the Mach 10 shock; consult [31] for a detailed discussion of this problem.

Two grids were used in our simulations: $480 \times 120$ and $960 \times 240$. In Figure 5.7 we plot the results obtained by NT-UNO-S and NT-UNO-N, zooming on $[0,3] \times[0,1]$. Only the density is shown for the coarser grid. The entropy is defined here as $\frac{P}{\rho^{\gamma}}$. We observe that the MM2 limiter performs very similarly to the UNO limiter, whereas the MM1 limiter is too dissipative for this problem.

6. Conclusions. We applied the nonstaggered schemes to several canonical problems and compared the numerical results with the staggered version of these schemes. In general, the nonstaggered schemes produce slightly lower resolution for shocks than the staggered schemes. We recommend that, whenever possible, the UNO limiter be used for robustness and accuracy. The most expensive test, the double Mach reflection problem on the $960 \times 240$ grid, took only about 8 minutes on a Cray C-90.

Acknowledgment. D.L. would also like to thank the Harten fellowship for its support.

\section{REFERENCES}

[1] P. Arminijon, D. Stanescu, and M.-C. Viallon, A two-dimensional finite volume extension of the Lax-Friedrichs and Nessyahu-Tadmor schemes for compressible flow, in Proc. 6th Int. Sympos. on CFD, Lake Tahoe, vol. 4, M. Hafez, ed., 1995, pp. 7-14.

[2] K. O. Friedrichs AND P. D. Lax, Systems of conservation equations with a convex extension, Proc. Nat. Acad. Sci. U.S.A., 68 (1971), pp. 1686-1688.

[3] S. K. Godunov, A finite difference method for the numerical computation of discontinuous solutions of the equations of fluid dynamics, Mat. Sb., 47 (1959), pp. 271-290.

[4] E. Godlewski and P.-A. Raviart, Hyperbolic Systems of Conservation Laws, Mathematics Appl., Ellipses, Paris, 1991.

[5] A. Harten, High resolution schemes for hyperbolic conservation laws, J. Comput. Phys., 49 (1983), pp. 357-393.

[6] A. Harten and S. Osher, Uniformly high order accurate nonoscillatory schemes. I, SIAM J. Numer. Anal., 24 (1982), pp. 279-309.

[7] H. T. HuYnh, A piecewise-parabolic dual-mesh method for the Euler equations, AIAA-95-1739CP, in 12th AIAA CFD Conf., 1995.

[8] G.-S. Jiang And C.-W. Shu, Efficient implementation of weighted ENO schemes, J. Comput. Phys., 126 (1996), pp. 202-228.

[9] G.-S. Jiang AND E. TADMOR, Nonoscillatory central schemes for multidimensional hyperbolic conservation laws, SIAM J. Sci. Comput., 19 (1998), pp. 1892-1917.

[10] R. Kupferman, A numerical study of the axisymmetric Couette-Taylor problem using a fast high-resolution second-order central scheme, SIAM J. Sci. Comput., 20 (1998), pp. 858877.

[11] R. Kupferman And E. TAdmor, A fast high-resolution second-order central scheme for incompressible flows, Proc. Nat. Acad. Sci. U.S.A., 94 (1997), pp. 4848-4852.

[12] P. D. LAx, Weak solutions of non-nonlinear hyperbolic equations and their numerical computations, Comm. Pure Appl. Math., 7 (1954), pp. 159-193.

[13] B. VAN LEER, Towards the ultimate conservative difference scheme, V. A second-order sequel to Godunov's method, J. Comput. Phys., 32 (1979), pp. 101-136.

[14] R. J. LeVEque, High-resolution conservative algorithms for advection in incompressible flow, SIAM J. Numer. Anal., 33 (1996), pp. 627-665. 
[15] R. J. LeVeque, Numerical Methods for Conservation Laws, Lectures in Mathematics, Birkhauser Verlag, Basel, 1992

[16] D. Levy, Third-Order 2D Central Schemes for Hyperbolic Conservation Laws, preprint.

[17] D. Levy AND E. TADmor, Non-oscillatory central schemes for the incompressible 2-D Euler equations, Math. Res. Lett., 4 (1997), pp. 1-20.

[18] D. LEVy AND E. TADMOR, Non-oscillatory boundary treatment for staggered central schemes, submitted.

[19] X.-D. LIU AND S. OSHER, Nonoscillatory high order accurate self-similar maximum principle satisfying shock capturing schemes I, SIAM J. Numer. Anal., 33 (1996), pp. 760-779.

[20] X.-D. LiU AND S. Osher, Convex ENO high order multi-dimensional schemes without field by field decomposition or staggered grids, J. Comput. Phys., 142 (1998), pp. 304-330.

[21] X.-D. LIU AND E. TADMOR, Third order nonoscillatory central scheme for hyperbolic conservation laws, Numer. Math., 79 (1998), pp. 397-425.

[22] H. Nessyahu and E. TAdmor, Non-oscillatory central differencing for hyperbolic conservation laws, J. Comput. Phys., 87 (1990), pp. 408-463.

[23] S. OShER AND E. TADMOR, On the convergence of difference approximations to scalar conservation laws, Math. Comp., 50 (1988), pp. 19-51.

[24] P. L. RoE, Approximate Riemann solvers, parameter vectors, and difference schemes, J. Comput. Phys., 43 (1981), pp. 357-372.

[25] R. SANDers AND A. WeIser, A high resolution staggered mesh approach for nonlinear hyperbolic systems of conservation laws, J. Comput. Phys., 101 (1992), pp. 314-329.

[26] C. W. Shu And S. Osher, Efficient implementation of essentially nonoscillatory schemes, II, J. Comput. Phys., 83 (1989), pp. 32-78.

[27] G. SoD, A survey of several finite difference methods for systems of nonlinear hyperbolic conservation laws, J. Comput. Phys., 22 (1978), pp. 1-31.

[28] P. K. Sweby, High resolution schemes using flux limiters for hyperbolic conservation laws, SIAM J. Numer. Anal., 21 (1984), pp. 995-1011.

[29] E. TADMOR, Numerical viscosity and the entropy condition for conservative difference schemes, Math. Comp., 43 (1984), pp. 369-381.

[30] E. TADMOR AND C. C. WU, Central scheme for the multidimensional MHD equations, in preparation.

[31] P. Woodward and P. Colella, The numerical simulation of two-dimensional fluid flow with strong shocks, J. Comput. Phys., 54 (1988), pp. 115-173. 